\title{
Cramér-Rao Bounds for Coprime and Other Sparse Arrays, which Find More Sources than Sensors ${ }^{\text {th }}$
}

\author{
Chun-Lin Liu, P. P. Vaidyanathan \\ Dept. of Electrical Engineering, MC 136-93, California Institute of Technology, Pasadena, CA 91125, USA
}

\begin{abstract}
The Cramér-Rao bound (CRB) offers a lower bound on the variances of unbiased estimates of parameters, e.g., directions of arrival (DOA) in array processing. While there exist landmark papers on the study of the CRB in the context of array processing, the closed-form expressions available in the literature are not easy to use in the context of sparse arrays (such as minimum redundancy arrays (MRAs), nested arrays, or coprime arrays) for which the number of identifiable sources $D$ exceeds the number of sensors $N$. Under such situations, the existing literature does not spell out the conditions under which the Fisher information matrix is nonsingular, or the condition under which specific closed-form expressions for the CRB remain valid. This paper derives a new expression for the CRB to fill this gap. The conditions for validity of this expression are expressed as the rank condition of a matrix defined based on the difference coarray. The rank condition and the closed-form expression lead to a number of new insights. For example, it is possible to prove the previously known experimental observation that, when there are more sources than sensors, the CRB stagnates to a constant value as the SNR tends to infinity. It is also possible to precisely specify the relation between the number of sensors and the number of uncorrelated sources such that these conditions are valid. In particular, for nested arrays, coprime arrays, and MRAs, the new expressions remain valid for $D=O\left(N^{2}\right)$, the precise detail depending on the specific array geometry.
\end{abstract}

Keywords: Cramér-Rao bounds, Fisher information matrix, sparse arrays, coprime arrays, difference coarrays.

\section{Introduction}

The celebrated Cramér-Rao bound (CRB), which has influenced our thinking for many decades of statistical signal processing, has found significant use in direction-of-arrival (DOA) problems, among others $[20,42,59,78,102]$. The DOA problem is of great importance in passive array processing [102], radar [26, 43, 87], digital communications [25], and radio astronomy [33], to name a few [34, 46, 95]. The CRB offers a lower bound on the variances of unbiased estimates of the parameters (e.g., DOA). Closed-form expressions for the $\mathrm{CRB}$ offer insights into the dependence of the array performance with respect to various parameters such as the number of sensors $N$ in the array, the array geometry, the number of sources $D$, the number of snapshots, signal to noise ratio (SNR), and so forth.

Two of the most influential papers in the DOA context are the papers by Stoica and Nehorai [90] and [91]. These papers distinguish between the deterministic CRB and the stochastic CRB (reviewed here in Section 3), and obtain closed-form expressions for these. In both cases, the expressions for CRB come from the inversion of the

\footnotetext{
This work was supported in parts by the ONR grant N00014-151-2118, and the California Institute of Technology.

Email addresses: cl.liu@caltech.edu (Chun-Lin Liu), ppvnath@systems. caltech.edu (P. P. Vaidyanathan)
}

Fisher information matrix (FIM), which contains information about all the unknown parameters. An appropriate principal submatrix of this inverse reveals the CRB of the DOAs, which we denote as $\operatorname{CRB}(\overline{\boldsymbol{\theta}})$. In this paper, we will be especially interested in the stochastic CRB because the model assumptions used therein are more appropriate in our context, namely sparse array processing using the difference coarray (Section 3).

The specific CRB expressions given in Eqs. (2.11) and (3.1) of [91] are valid only when $D<N$ (fewer sources than sensors). This is because the expressions are based on the inverse of the matrix $\mathbf{A}^{H} \mathbf{A}$ (e.g., see the equation after (2.11) in [91]), where $\mathbf{A}$ is the so-called array manifold matrix (see Section 2.1). The assumption $D<N$ is however not fundamental to the existence of CRB of the DOA parameters because even when $D \geq N$, with proper prior information, the FIM can remain nonsingular (invertible) under a much broader range of conditions, as we shall prove in this paper. So it is possible to get more useful expressions which do not involve $\left(\mathbf{A}^{H} \mathbf{A}\right)^{-1}$.

The closed-form expressions for $\operatorname{CRB}(\overline{\boldsymbol{\theta}})$ given in Eq. (3.1) of [91] assume an arbitrary covariance matrix for the sources. For the case where it is known a priori that the sources are uncorrelated, it has been shown in a very interesting paper by Jansson et al. [40] that the CRB is in general smaller than what one would get if one substituted a diagonal covariance in the expression (3.1) given in [91].

April 12, 2016 
Closed-form expressions for $\operatorname{CRB}(\overline{\boldsymbol{\theta}})$ for the case of uncorrelated sources are also given in [40]. The authors mention that it is possible to estimate more sources than sensors in the case of certain array configurations [40, 68]. However, the detailed conditions under which this is possible are not provided in [40]. Furthermore, all the examples given in [40] are for the case of uniform linear arrays (ULAs), for which the number of identifiable sources is less than the number of sensors. Finally, the inverses of certain matrices are assumed to exist in the CRB expression (13) in [40] although the conditions under which these inverses exist are not spelt out. In fact, the appearance of $m-d$ (i.e., $N-D$ in our notation) in the denominator Eq. (14) of [40] makes this expression invalid when $N=D$.

Most importantly, suppose we are interested in the question "Given an array configuration, what is the upper limit on the number of sources $D$ (in relation to the number of sensors) such that the expression for $\operatorname{CRB}(\overline{\boldsymbol{\theta}})$ is valid?" This is not answered in [40]. One of our contributions here is to give a precise theoretical answer to such questions. The reason why this is possible is because there are multiple ways to express the CRB in closed forms, and some of them are more revealing than others.

The reason for the renewed interest in finding more useful closed-form expressions for the CRB is the following. For a long time, sparse arrays, such as the minimum redundancy arrays (MRAs) have been known to be able to identify more sources than sensors $(D \geq N)[61]$. More recently, the development of sparse arrays such as the nested arrays [63], the coprime arrays [64, 98], and their extensions $[51,52,54,75,76]$, have generated a new wave of interest in this topic. These new arrays have simple closedform expressions for array geometry (compared to MRAs which do not have this advantage), which makes them more practical than MRAs. The most essential property of these successful sparse arrays is that, given $N$ sensors, the difference coarrays of these arrays have $O\left(N^{2}\right)$ elements, which allows them to identify $D=O\left(N^{2}\right)$ sources using $N$ sensors. In particular, therefore, $D \gg N$ is possible as demonstrated amply in $[1-3,16-19,21,28-$ $32,36,40,53,57,58,61,63,64,67,68,70,71,74-$ 77, 93, 94, 96, 98, 107, 108].

It is therefore of great importance to study the performance limits of these sparse arrays by using standard tools such as the CRB. If we try to do this using the existing results in the literature, we run into a road block. Either the known closed-form expressions are not valid when $D \geq N$, or the precise conditions under which they are valid are not specified. In this context, it is worth mentioning that the pioneering work by Abramovich et al. many years ago [1] discussed the performances of MRAs by successfully plotting the CRB even for the case of $D \geq N$. The same can be done today for nested and coprime arrays. However, the theoretical conditions under which the CRB exists (for the case $D \geq N$ ) have not been spelt out in the past.

We now summarize the main contributions of our paper. Starting from the Fisher information matrix for the case of stochastic CRB with uncorrelated priors, as in [40], we derive a new closed-form expression for the CRB, specifically for the case of uncorrelated sources. The new CRB expressions are valid if and only if the FIM is nonsingular. The condition for the validity of our CRB expression are here expressed explicitly in terms of the geometry of the difference coarray. Thus, with $\mathbb{D}$ denoting the difference coarray of a linear array, let $\mathbf{V}_{\mathbb{D}}$ be the array manifold matrix defined on this difference coarray. So $\mathbf{V}_{\mathbb{D}}$ is a $|\mathbb{D}| \times D$ matrix. The number of rows is the size of difference coarray (which could be $O\left(N^{2}\right)$ ), and the number of columns is the number of sources. Now consider the $|\mathbb{D}| \times(2 D+1)$ matrix

$$
\mathbf{A}_{c}=\left[\operatorname{diag}(\mathbb{D}) \mathbf{V}_{\mathbb{D}} \quad \mathbf{V}_{\mathbb{D}} \quad \mathbf{e}_{0}\right]
$$

where $\operatorname{diag}(\mathbb{D})$ is a diagonal matrix with $\mathbb{D}$ on its diagonals, and $\mathbf{e}_{0}$ is an all zero vector except for one nonzero element, the location of which depends on the coarray geometry. We refer to the matrix (1) as the augmented coarray manifold matrix or ACM matrix. The main result is that the FIM is nonsingular if and only if the ACM matrix $\mathbf{A}_{c}$ has full column rank $2 D+1$. We shall refer to this as the rank condition. To the best of our knowledge, the invertibility of the FIM has not in the past been characterized in terms of the difference coarray geometry. The proposed CRB expression holds under this rank condition, and is given by our Eq. (49) (to be proved in Theorem 2). Thus the specific CRB expression is valid whenever the FIM is invertible.

The invertibility of FIM, expressed as a rank condition on the ACM matrix, leads to a number of further insights as we shall elaborate in the paper. In short, the rank condition depends explicitly only on the difference coarray and the DOAs, whereas the CRB itself depends also on the physical array, the number of snapshots, and the SNR (Properties 3 and 4 of Section 5). We will also see that if the rank condition on the ACM matrix is satisfied, then $\operatorname{CRB}(\overline{\boldsymbol{\theta}})$ converges to zero as the number of snapshots increases. This is true even for the case $D \geq N$ (more sources than sensors).

Rather surprisingly, the same cannot be said for the SNR. Thus, when the array manifold matrix $\mathbf{V}_{\mathbb{S}}$ has full row rank, implying $D \geq N$, we show that if the sources have identical powers $p$, then for a fixed number of snapshots, the CRB stagnates to a constant value as $p / p_{n}$ goes to infinity, where $p_{n}$ is the noise power (Theorem 4 in Section 5). When $\mathbf{V}_{\mathbb{S}}$ does not have full row rank, we will see that the CRB decays to zero for large SNR, under suitable assumptions (Theorem 3 in Section 5). Similar behavior for $D \geq N$ and $D<N$ was first noticed by Abramovich et al. in [1] experimentally. Here we elaborate the conditions and find these to be provable consequences of the specific CRB expression we derive.

Another corollary of our results is that if the central ULA segment in the difference coarray has length $L$, then the rank condition on the ACM matrix is indeed satisfied 
as long as $D \leq(L-1) / 2$ (Theorem 5$)$. This is very satisfying because, experimentally it has indeed been observed repeatedly that, methods such as spatial-smoothing based coarray MUSIC always succeed in identifying the sources in this case [53, 63, 64, 98].

Yet another outcome of the rank condition is that it is possible to give a precise bound on the number of sources $D$ such that the proposed CRB expression is valid. In particular, for nested arrays, coprime arrays, and MRAs, the FIM is provably invertible for $O\left(N^{2}\right)$ uncorrelated sources (the exact number depending on the specific array used, the source locations, and so forth), and therefore the CRB expression is provably valid for this many sources. Needless to say, our results (the rank condition and the proposed CRB expression) and the conclusions derived from them are valid for any linear array, sparse or otherwise, including the redoubtable ULA.

Paper outline. In Section 2, we introduce the data model and provide a brief review of sparse arrays (minimum redundancy arrays, minimum hole arrays, nested arrays, coprime arrays, and so on). In Section 3, we review some known results on the CRB, which are necessary for building up new results. The new CRB expression and the rank condition for its validity are presented in Section 4. The implications of this CRB expression are detailed in Section 5 and 6. Numerical examples are given in Section 7 to demonstrate the implications of the new results. Details of some of the proofs are given in Appendix A to Appendix D.

\subsection{Notation}

Scalars, vectors, matrices, and sets are denoted by lowercase letters $(a)$, lower-case letters in bold face (a), uppercase letters in bold face $(\mathbf{A})$, and upper-case letters in blackboard boldface $(\mathbb{A}) .[\mathbf{A}]_{i, j}$ indicates the $(i, j)$ th entry of $\mathbf{A}$. The complex conjugate, the transpose, and the complex conjugate transpose of $\mathbf{A}$ are $\mathbf{A}^{*}, \mathbf{A}^{T}$, and $\mathbf{A}^{H}$, respectively. Letting $\mathbf{A} \in \mathbb{C}^{M \times N}$, the Kronecker product between $\mathbf{A}$ and $\mathbf{B}$ is defined as

$$
\mathbf{A} \otimes \mathbf{B}=\left[\begin{array}{cccc}
{[\mathbf{A}]_{1,1} \mathbf{B}} & {[\mathbf{A}]_{1,2} \mathbf{B}} & \ldots & {[\mathbf{A}]_{1, N} \mathbf{B}} \\
{[\mathbf{A}]_{2,1} \mathbf{B}} & {[\mathbf{A}]_{2,2} \mathbf{B}} & \ldots & {[\mathbf{A}]_{2, N} \mathbf{B}} \\
\vdots & \vdots & \ddots & \vdots \\
{[\mathbf{A}]_{M, 1} \mathbf{B}} & {[\mathbf{A}]_{M, 2} \mathbf{B}} & \ldots & {[\mathbf{A}]_{M, N} \mathbf{B}}
\end{array}\right]
$$

The Hadamard product between $\mathbf{A}$ and $\mathbf{B}$ of the same size is $\mathbf{A} \odot \mathbf{B}$ such that $[\mathbf{A} \odot \mathbf{B}]_{i, j}=[\mathbf{A}]_{i, j}[\mathbf{B}]_{i, j}$. The Khatri-Rao matrix product $\circ$ is defined as

$$
\begin{aligned}
& {\left[\begin{array}{llll}
\mathbf{a}_{1} & \mathbf{a}_{2} & \ldots & \mathbf{a}_{N}
\end{array}\right] \circ\left[\begin{array}{llll}
\mathbf{b}_{1} & \mathbf{b}_{2} & \ldots & \mathbf{b}_{N}
\end{array}\right] } \\
= & {\left[\begin{array}{llll}
\mathbf{a}_{1} \otimes \mathbf{b}_{1} & \mathbf{a}_{2} \otimes \mathbf{b}_{2} & \ldots & \mathbf{a}_{N} \otimes \mathbf{b}_{N}
\end{array}\right] . }
\end{aligned}
$$

For a full column rank matrix $\mathbf{A}$, the matrices

$$
\begin{aligned}
\boldsymbol{\Pi}_{\mathbf{A}} & =\mathbf{A}\left(\mathbf{A}^{H} \mathbf{A}\right)^{-1} \mathbf{A}^{H} . \\
\boldsymbol{\Pi}_{\mathbf{A}}^{\perp} & =\mathbf{I}-\mathbf{A}\left(\mathbf{A}^{H} \mathbf{A}\right)^{-1} \mathbf{A}^{H} .
\end{aligned}
$$

denote the orthogonal projection onto the column space of $\mathbf{A}$, and to the null space of $\mathbf{A}^{H}$, respectively. $\operatorname{diag}\left(a_{1}, \ldots, a_{n}\right)$ is a diagonal matrix with diagonal entries $a_{1}, \ldots, a_{n}$. For a real set $\mathbb{A}=\left\{a_{1}, \ldots, a_{n}\right\}$ such that $a_{1}<$ $\cdots<a_{n}, \operatorname{diag}(\mathbb{A})=\operatorname{diag}\left(a_{1}, \ldots, a_{n}\right) \cdot \operatorname{rank}(\mathbf{A})$ is the rank of $\mathbf{A} . \operatorname{tr}(\mathbf{A})$ denotes the trace of $\mathbf{A}$, which is the sum of diagonal entries. $\operatorname{vec}\left(\left[\mathbf{a}_{1}, \mathbf{a}_{2}, \ldots, \mathbf{a}_{N}\right]\right)=\left[\mathbf{a}_{1}^{T}, \mathbf{a}_{2}^{T}, \ldots, \mathbf{a}_{N}^{T}\right]^{T}$ is the vectorization operation. The cardinality of a set $\mathbb{A}$ is denoted as $|\mathbb{A}|$. $\mathbb{E}[\cdot]$ denotes the expectation operator. $\mathcal{N}(\boldsymbol{\mu}, \mathbf{C})$ is a multivariate real-valued normal distribution with mean $\boldsymbol{\mu}$ and covariance $\mathbf{C}$. $\mathcal{C N}(\mathbf{m}, \boldsymbol{\Sigma})$ is a complex normal distribution with mean $\mathbf{m}$ and covariance matrix $\Sigma$.

Let $\mathbb{S}$ be an integer set and let the signal defined over $\mathbb{S}$ denoted by a column vector $\mathbf{x}_{\mathbb{S}}$. The square bracket notation $\left[\mathbf{x}_{\mathbb{S}}\right]_{i}$ represents the $i$ th component of $\mathbf{x}_{\mathbb{S}}$. The triangular bracket notation $\left\langle\mathbf{x}_{\mathbb{S}}\right\rangle_{n}$ denotes the signal value on the support $n \in \mathbb{S}$ and is very useful for nonuniform arrays. For instance, if $\mathbb{S}=\{0,2,5\}$ and $\mathbf{x}_{\mathbb{S}}=[-1,1,4]^{T}$, then the square brackets $\left[\mathbf{x}_{\mathbb{S}}\right]_{1}=-1,\left[\mathbf{x}_{\mathbb{S}}\right]_{2}=1,\left[\mathbf{x}_{\mathbb{S}}\right]_{3}=$ 4 , and the triangular brackets $\left\langle\mathbf{x}_{\mathbb{S}}\right\rangle_{0}=-1,\left\langle\mathbf{x}_{\mathbb{S}}\right\rangle_{2}=1$, $\left\langle\mathbf{x}_{\mathbb{S}}\right\rangle_{5}=4$.

\section{The Data Model and Sparse Arrays}

\subsection{The data model}

In sensor array processing, the sensor locations $n d$ are described by an integer set $\mathbb{S}$ such that $n \in \mathbb{S}$, and $d=\lambda / 2$ is half of the wavelength. We assume that this sensor array $\mathbb{S}$ is illuminated by $D$ monochromatic plane waves with DOA $\theta_{i}$ satisfying $-\pi / 2 \leq \theta_{i}<\pi / 2$ for $i=1,2, \ldots, D$. Then, the measurements on the sensor array $\mathbb{S}$, denoted by $\mathbf{x}_{\mathbb{S}}$, can be modeled as $[33,60,102]$

$$
\mathbf{x}_{\mathbb{S}}=\sum_{i=1}^{D} A_{i} \mathbf{v}_{\mathbb{S}}\left(\bar{\theta}_{i}\right)+\mathbf{n}_{\mathbb{S}} \quad \in \mathbb{C}^{|\mathbb{S}|}
$$

where $A_{i}$ and $\bar{\theta}_{i}=(d / \lambda) \sin \theta_{i}$ represent the complex amplitude and the normalized DOA of the $i$ th source. $\mathbf{v}_{\mathbb{S}}\left(\bar{\theta}_{i}\right)=\left[e^{j 2 \pi \bar{\theta}_{i} n}\right]_{n \in \mathbb{S}}$ is the steering vector defined on $\mathbb{S}$. $\mathbf{n}_{\mathbb{S}}$ is a random noise term.

To analyze the DOA estimation performance based on $K$ independent and identically distributed snapshots of (4), two probability models are commonly considered:

1. The conditional or deterministic model [90]: The complex amplitude $\left[A_{i}\right]_{i=1}^{D}$ is assumed to be unknown but non-random. The noise vector $\mathbf{n}_{\mathbb{S}}$ is a complex Gaussian random vector with mean zero and covariance $p_{n} \mathbf{I}$.

2. The unconditional or stochastic model [91]: $\left[A_{i}\right]_{i=1}^{D}$ is assumed to be a Gaussian random vector with mean zero and covariance $\mathbf{P}$ such that $[\mathbf{P}]_{i, j}=\mathbb{E}\left[A_{i} A_{j}^{*}\right]$. $\mathbf{n}_{\mathbb{S}}$ is a complex Gaussian random vector with mean zero and covariance $p_{n} \mathbf{I}$. $\mathbf{n}_{\mathbb{S}}$ and $A_{i}$ are uncorrelated.

A more detailed description of these models can be found in [89-91]. 


\subsection{Review of coarray-based DOA estimators}

DOA estimators aim to evaluate the normalized DOAs $\overline{\boldsymbol{\theta}}$ based on the sensor output $\mathbf{x}_{\mathbb{S}}$. This goal can be achieved by a variety of DOA estimators, including the Barlett beamformer [8], the Capon beamformer [15], the MUSIC algorithm [10, 84], the ESPRIT algorithm [81], and many others $[6,7,24,27,62,69,72,79]$. However, the performance of these DOA estimators is limited by the number of sensors. For instance, it was shown in [90, 102] that for $N$-sensor ULA, the MUSIC algorithm identifies at most $N-1$ sources.

For the case where the sources are known to be uncorrelated and the array is the ULA, some improved DOA estimators have been reported [11, 40, 41, 49, 104, 105]. Furthermore, with uncorrelated priors and suitable array configurations, coarray-based DOA estimators convert the sample covariance matrix of the sensor outputs into the so-called difference coarray domain, using which DOAs are estimated $[1,2,18,19,21,31,35,53,55,57,58,63-$ $67,70,71,74,93,96,107]$. For appropriate array geometries, the size of the difference coarray is much larger than the number of sensors, which makes it possible to identify more sources than the number of sensors.

The details of coarray-based DOA estimators are developed as follows. It is assumed that the sources are uncorrelated and the sensor output $\mathbf{x}_{\mathbb{S}}$ follows the stochastic model, as in (4), where the complex amplitude $\left[A_{i}\right]_{i=1}^{D}$ and the noise term $\mathbf{n}_{\mathbb{S}}$ have the following distribution:

$$
\begin{aligned}
{\left[A_{1}, A_{2}, \ldots, A_{D}\right]^{T} } & \sim \mathcal{C N}\left(\mathbf{0}, \operatorname{diag}\left(p_{1}, p_{2}, \ldots, p_{D}\right)\right) \\
\mathbf{n}_{\mathbb{S}} & \sim \mathcal{C N}\left(\mathbf{0}, p_{n} \mathbf{I}\right)
\end{aligned}
$$

where $p_{1}, p_{2}, \ldots, p_{D}>0$ are the source powers and $p_{n}>0$ is the noise power. It is assumed that sources are uncorrelated to noise, namely, $\mathbb{E}\left[A_{i} \mathbf{n}_{\mathbb{S}}^{H}\right]=\mathbf{0}$. Under these assumptions, the covariance matrix of $\mathbf{x}_{\mathbb{S}}$ is given by

$$
\mathbf{R}_{\mathbb{S}}=\mathbb{E}\left[\mathbf{x}_{\mathbb{S}} \mathbf{x}_{\mathbb{S}}^{H}\right]=\sum_{i=1}^{D} p_{i} \mathbf{v}_{\mathbb{S}}\left(\bar{\theta}_{i}\right) \mathbf{v}_{\mathbb{S}}^{H}\left(\bar{\theta}_{i}\right)+p_{n} \mathbf{I} .
$$

Vectorizing (7) and removing duplicated entries give [53, $57,58,63,64]$

$$
\mathbf{x}_{\mathbb{D}}=\sum_{i=1}^{D} p_{i} \mathbf{v}_{\mathbb{D}}\left(\bar{\theta}_{i}\right)+p_{n} \mathbf{e}_{0}
$$

where $\mathbf{e}_{0}$ is a column vector satisfying $\left\langle\mathbf{e}_{0}\right\rangle_{m}=\delta_{m, 0}$. Here $\mathbf{x}_{\mathbb{D}}$ can be regarded as a deterministic data vector on the difference coarray $\mathbb{D}$, which is defined as

Definition 1 (Difference coarray $\mathbb{D})$. Let $\mathbb{S}$ be an integer set defining the sensor locations. The difference set is defined as $\mathbb{D}=\left\{n_{1}-n_{2} \mid n_{1}, n_{2} \in \mathbb{S}\right\}$.

The difference coarray is symmetric, i.e., if $m \in \mathbb{D}$, then $-m \in \mathbb{D}$, so we often show the non-negative part only. It is also useful to characterize the contiguous ULA section in the difference coarray, denoted as $\mathbb{U}$, which is utilized in many coarray-based DOA estimators:

Definition 2 (U, the central ULA segment). Let $\mathbb{D}$ be the difference coarray of $\mathbb{S}$ and let $m$ be the largest integer such that $\{0, \pm 1, . . \pm m\} \subseteq \mathbb{D}$. Then $\mathbb{U}=\{0, \pm 1, . . \pm m\}$ is called the central ULA segment of $\mathbb{D}$.

Definition $3(w(m)$, the weight function). Let $\mathbb{S}$ be the physical array and $\mathbb{D}$ be its difference coarray. The weight function is the number of sensor pairs with separation $m$, defined as

$$
\begin{aligned}
w(m) & =|\mathbb{M}(m)|, \\
\mathbb{M}(m) & =\left\{\left(n_{1}, n_{2}\right) \in \mathbb{S}^{2} \mid n_{1}-n_{2}=m\right\} .
\end{aligned}
$$

For example, if $\mathbb{S}=\{0,1,4\}$, then the difference coarray $\mathbb{D}=\{-4,-3,-1,0,1,3,4\}$ while the set $\mathbb{U}=\{-1,0,1\}$. The weight function $w(m)$ satisfies $w(0)=3, w( \pm 1)=$ $w( \pm 3)=w( \pm 4)=1$, and $w(m)=0$ for other $m$ 's. It can be seen directly from Definition 2 that $\mathbb{U} \subseteq \mathbb{D}$.

Coarray-based DOA estimators are based on the finitesnapshot version of (7) and (8). Assume that $\widetilde{\mathbf{x}}_{\mathbb{S}}(k)$ for $k=1,2, \ldots, K$ denote $K$ snapshots of $\mathbf{x}_{\mathbb{S}}$. The sample covariance matrix is

$$
\widetilde{\mathbf{R}}_{\mathbb{S}}=\frac{1}{K} \sum_{k=1}^{K} \widetilde{\mathbf{x}}_{\mathbb{S}}(k) \widetilde{\mathbf{x}}_{\mathbb{S}}^{H}(k) .
$$

The finite-snapshot version of $\mathbf{x}_{\mathbb{D}}$, denoted by $\widetilde{\mathbf{x}}_{\mathbb{D}}$, can be constructed from (11) as follows [53]:

$$
\left\langle\widetilde{\mathbf{x}}_{\mathbb{D}}\right\rangle_{m}=\frac{1}{w(m)} \sum_{\left(n_{1}, n_{2}\right) \in \mathbb{M}(m)}\left\langle\widetilde{\mathbf{R}}_{\mathbb{S}}\right\rangle_{n_{1}, n_{2}}, \quad m \in \mathbb{D},
$$

where $w(m)$ and $\mathbb{M}(m)$ are defined in Definition 3 .

The signal $\widetilde{\mathbf{x}}_{\mathbb{D}}$ on the difference coarray $\mathbb{D}$ facilitates a variety of DOA estimators. For instance, the augmented covariance matrix method [70, 71] constructs an augmented covariance matrix $\widetilde{\mathbf{R}}_{\text {aug }}$, which has larger dimension than $\widetilde{\mathbf{R}}_{\mathbb{S}}$, from $\widetilde{\mathbf{x}}_{\mathbb{D}}$. The spatially smoothed MUSIC algorithm (SS MUSIC) [63, 64] evaluates a spatially smoothed matrix $\widetilde{\mathbf{R}}_{s s}$ based on $\widetilde{\mathbf{x}}_{\mathbb{D}}$. Another family is the sparsity-based methods $[67,93,107]$, which relate $\widetilde{\mathbf{x}}_{\mathbb{D}}$ to $(8)$ using overcomplete dictionaries and enforce sparsity constraints to source profiles.

It is known that, in SS MUSIC, the size and the structure of the difference coarray are crucial to the number of identifiable sources for nested arrays [63] and coprime arrays [64]. Empirically, the number of identifiable sources can be characterized by the following properties:

Property 1 ([63, 64]). If the number of distinct sources $D \leq(|\mathbb{U}|-1) / 2$, then SS MUSIC is able to identify these sources for larger number of snapshots.

Property $2([66])$. If $D>(|\mathbb{D}|-1) / 2$, then it is impossible to identify these sources using SS MUSIC. 


\subsection{Review of sparse array design}

Property 1 and 2 indicate that the size of $\mathbb{D}$ and $\mathbb{U}$ plays a crucial role in the number of identifiable sources. So it is motivating to design the sensor locations $\mathbb{S}$ such that $|\mathbb{D}|$ or $|\mathbb{U}|$ is large. Several well-known solutions include minimum redundancy arrays (MRA) [61], minimum hole arrays (MHA) [94, 103], nested arrays [63], coprime arrays [98], super nested arrays $[51,52,54]$, and many other variants $[4,9,12-14,23,38,39,50,66,76,82,83,85,86,106]$. All of them have $O\left(N^{2}\right)$ distinct lags in the difference coarray, given $O(N)$ physical sensors.

Minimum redundancy arrays (MRA) [61] maximize the coarray size under the constraint that the difference coarray consists of consecutive integers ${ }^{1}$. In particular, assume that $N$ distinct sensors are located at $s_{1}, s_{2}, \ldots, s_{N}$ such that $s_{1}<s_{2}<\cdots<s_{N}$. Let $A=s_{N}-s_{1}$ be the array aperture. The $N$-sensor MRAs solve the following optimization problem:

Objective: Maximize the array aperture $A$ subject to the following constraint: the difference coarray $\mathbb{D}$ should contain all the integers from $-A$ to $A$.

As a example, Fig. 1(a) illustrates a 6-sensor MRA, its difference coarrary $\mathbb{D}$, and the associated weight functions. It can be seen that these sensors are placed non-uniformly along a straight line while the difference coarray contains consecutive integers from -13 to 13 . The size of the difference coarray is 27 , which is much larger than the number of sensors, 6. The main drawback of MRA is that the sensor locations cannot be expressed in closed forms for large $N$ and can only be evaluated by searching algorithms $[39,48,50,83]$.

Minimum hole arrays (MHA) [94, 103], which are also named as Golomb arrays or minimum gap arrays, minimize the number of holes in the coarray domain. More specifically, let $s_{1}, s_{2}, \ldots, s_{N}$ be $N$ sensor locations such that $s_{1}<s_{2}<\cdots<s_{N}$, and $A=s_{N}-s_{1}$ be the array aperture. The "holes" are defined as the set of integers $h$ such that $1 \leq h<A$ and $h$ does not belong to the different coarray $\mathbb{D}$. Then, the MHAs with $N$ sensors are solutions to the following optimization problem:

Objective: Minimize the number of the holes subject to the following constraint: the weight function $w(m)$ is either 0 or 1 for $1 \leq|m| \leq A$.

Note that $w(m)=0$ corresponds to holes and $w(m)=1$ means that the difference $m$ occurs exactly once. Thus the constraint ensures that no difference $m$ occurs more than once. For instance, Fig. 1(b) depicts the physical array and the difference coarray of a 6 -sensor MHA. It can be seen that in this case, $\mathbb{S}=\{0,1,4,10,12,17\}$ and the non-negative part of $\mathbb{D}$ is $\{0,1, \ldots, 13,16,17\}$. The set of holes is $\{14,15\}$. It can be verified that Fig. 1(b)

${ }^{1}$ This array configuration was denoted as restricted $M R A$ in [61]. satisfies the definition of MHA. Like MRA, the main issue for the MHA is that, there are no closed-form expressions for sensor locations [5, 22, 47, 80, 92, 94]. For further discussions, see [2] and the references therein.

Nested and coprime arrays $[63,98]$ are sparse arrays with simple geometries having closed-form expressions. Both have $O\left(N^{2}\right)$ distinct elements in the difference coarray domain, although they do not optimize the parameters that MRA or MHA seek to optimize. Nested arrays are composed of a dense ULA with sensor separation 1, and a sparse ULA with sensor separation $\left(N_{1}+1\right)$, in units of $\lambda / 2$. The closed-form sensor locations are given by [63]:

$$
\begin{aligned}
\mathbb{S}_{\text {nested }}=\left\{n \mid n=1,2, \ldots, N_{1}\right\} \\
\\
\quad \cup\left\{n\left(N_{1}+1\right) \mid n=1,2, \ldots, N_{2}\right\},
\end{aligned}
$$

where $N_{1}$ and $N_{2}$ are positive integers. Fig. 1(c) demonstrates a nested array with $N_{1}=N_{2}=3$. In this example, the number of physical sensors is 6 while the nonnegative part of the difference coarray consists of integers from 0 to 11. In particular, it was proved in [63] that, if $N_{1}$ is approximately $N_{2}$, then with $O(N)$ physical sensors, the size of the difference coarray is $O\left(N^{2}\right)$, which has the same order as MRA and MHA [61, 94]. One advantage of nested arrays is the simplicity of design equations for large number of elements [63], which cannot be achieved in MRA or MHA. Another advantage of nested arrays is that, the difference coarray consists of contiguous integers from $-N_{2}\left(N_{1}+1\right)+1$ to $N_{2}\left(N_{1}+1\right)-1$ and there are no holes. This property makes it possible to utilize the complete autocorrelation information in SS MUSIC [63].

Coprime arrays are another family of sparse arrays that enjoys long difference coarray and closed-form sensor locations [98]. They are composed of two sparse ULAs with sensor separations $M$ and $N$, respectively, in units of $\lambda / 2$. The set $\mathbb{S}$ is defined as follows:

$$
\begin{aligned}
\mathbb{S}_{\text {coprime }}=\{ & n M \mid n=0,1, \ldots, N-1\} \\
& \cup\{m N \mid m=1,2, \ldots, 2 M-1\},
\end{aligned}
$$

where $M$ and $N$ are a coprime pair of integers and $M<N$. Fig. 1(d) shows a coprime array with $M=2$ and $N=3$, as an example. The number of sensors is 6 and the nonnegative part of the difference coarray consists of consecutive integers from 0 to 7 but the lag 8 is missing. It was shown in $[64,98]$ that the difference coarray of coprime arrays, $\mathbb{D}_{\text {coprime, }}$ contains consecutive integers from $-M N$ to $M N$ and there are holes outside this region. It was further proved in $[76,108]$ that the maximum central contiguous ULA section of $\mathbb{D}_{\text {coprime, }}$, is actually from $-(M N+M-1)$ to $M N+M-1$. In other words, the size of the ULA segment in difference coarray for coprime arrays is $O(M N)$, given $O(M+N)$ physical sensors. The advantages of coprime arrays include, first, simple and closed-form sensor locations, as indicated in (13). Second, it was shown in $[51,64,98]$ that, compared to nested arrays, coprime arrays reduce the mutual coupling effect, which originates 


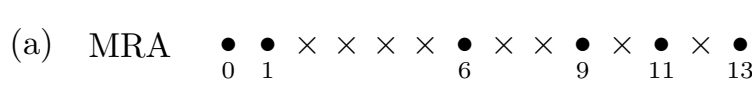

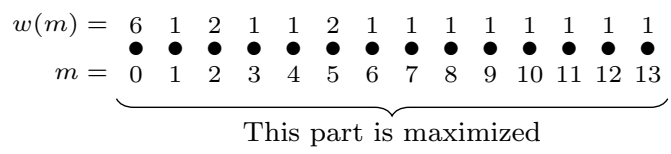

(b) MHA
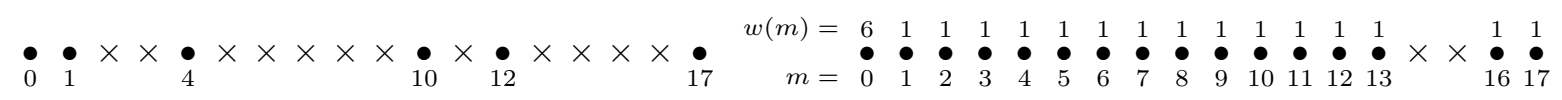

Dense ULA Sparse ULA

(c) Nested

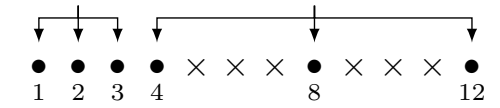

This part is minimized

Sparse ULA with separation $M$

(d)

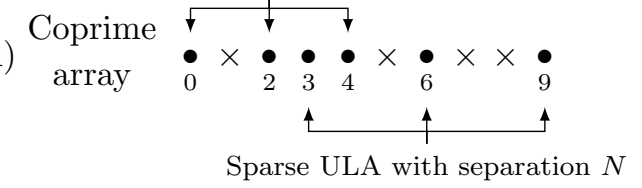

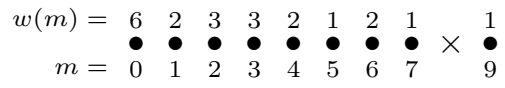

Figure 1: Array geometry of 6-sensor MRA, 6-sensor MHA, nested array with $N_{1}=N_{2}=3$, and coprime array with $M=2, N=3$. Bullets denote sensor locations in $\mathbb{S}$ or sensor separations in $\mathbb{D}$.

from the non-ideal interference between adjacent sensor outputs.

\section{Review of Cramér-Rao bounds}

Consider a real-valued random vector $\mathbf{x}$ with probability density function (pdf) $p(\mathbf{x} ; \boldsymbol{\alpha})$, where $\boldsymbol{\alpha}$ is a real-valued deterministic parameter vector. Assume that the $\operatorname{pdf} p(\mathbf{x} ; \boldsymbol{\alpha})$ satisfies the regularity condition $\mathbb{E}_{\mathbf{x}}[(\partial / \partial \boldsymbol{\alpha}) \log p(\mathbf{x} ; \boldsymbol{\alpha})]=$ $\mathbf{0}$, where $\mathbb{E}_{\mathbf{x}}[\cdot]$ indicates that the expectation is over $\mathbf{x}$. The Fisher information matrix (FIM) $\mathcal{I}(\boldsymbol{\alpha})$ is defined as

$$
[\mathcal{I}(\boldsymbol{\alpha})]_{i, j}=-\mathbb{E}_{\mathbf{x}}\left[\frac{\partial^{2}}{\partial[\boldsymbol{\alpha}]_{i} \partial[\boldsymbol{\alpha}]_{j}} \log p(\mathbf{x} ; \boldsymbol{\alpha})\right]
$$

It can be shown that the FIM is positive semidefinite [42]. Furthermore, if the FIM is positive definite, then the FIM is invertible and the Cramér-Rao bound (CRB) is given by the inverse of the FIM:

$$
\mathrm{CRB}(\boldsymbol{\alpha})=\mathcal{I}^{-1}(\boldsymbol{\alpha})
$$

which is also positive definite. The significance of the CRB is that the covariance of any unbiased estimator is lower bounded by the CRB. Namely, any unbiased estimator $\widehat{\boldsymbol{\alpha}}(\mathbf{x})$ for $\boldsymbol{\alpha}$, based on the observation $\mathbf{x}$, satisfies the following:

$$
\mathbb{E}_{\mathbf{x}}\left[\widehat{\boldsymbol{\alpha}}(\mathbf{x}) \widehat{\boldsymbol{\alpha}}(\mathbf{x})^{T}\right] \succeq \operatorname{CRB}(\boldsymbol{\alpha})
$$

where $\mathbf{A} \succeq \mathbf{B}$ is equivalent to $\mathbf{A}-\mathbf{B}$ being positive semidefinite for two Hermitian matrices $\mathbf{A}$ and $\mathbf{B}$. More details on the FIM and the CRB can be found in $[42,89,102]$.
For the deterministic or conditional CRB model, we use $K$ snapshots of (4):

$$
\mathbf{x}_{\mathbb{S}}(k)=\sum_{i=1}^{D} A_{i}(k) \mathbf{v}_{\mathbb{S}}\left(\bar{\theta}_{i}\right)+\mathbf{n}_{\mathbb{S}}(k), \quad k=1,2, \ldots, K
$$

It is assumed that the noise is both spatially and temporally uncorrelated, i.e., $\mathbb{E}\left[\mathbf{n}_{\mathbb{S}}\left(k_{1}\right) \mathbf{n}_{\mathbb{S}}^{H}\left(k_{2}\right)\right]=p_{n} \mathbf{I} \delta_{k_{1}, k_{2}}$, while the source amplitudes $A_{i}(k)$ are deterministic. As a result, the probability model for the deterministic model with $K$ snapshots becomes

$$
\left[\begin{array}{c}
\mathbf{x}_{\mathbb{S}}(1) \\
\mathbf{x}_{\mathbb{S}}(2) \\
\vdots \\
\mathbf{x}_{\mathbb{S}}(K)
\end{array}\right] \sim \mathcal{C N}\left(\left[\begin{array}{c}
\sum_{i=1}^{D} A_{i}(1) \mathbf{v}_{\mathbb{S}}\left(\bar{\theta}_{i}\right) \\
\sum_{i=1}^{D} A_{i}(2) \mathbf{v}_{\mathbb{S}}\left(\bar{\theta}_{i}\right) \\
\vdots \\
\sum_{i=1}^{D} A_{i}(K) \mathbf{v}_{\mathbb{S}}\left(\bar{\theta}_{i}\right)
\end{array}\right], p_{n} \mathbf{I}\right)
$$

and the parameters to be estimated contain normalized DOAs, source amplitudes at $K$ snapshots, and the noise power. In particular, the parameter vector $\boldsymbol{\alpha}_{\text {det }}$ for the deterministic model over $K$ snapshots becomes

$$
\boldsymbol{\alpha}_{\mathrm{det}}=\left[\bar{\theta}_{i}, \operatorname{Re}\left\{A_{i}(k)\right\}, \operatorname{Im}\left\{A_{i}(k)\right\}, p_{n}\right]^{T},
$$

where $1 \leq i \leq D, 1 \leq k \leq K$, and the subscript "det" stands for the deterministic model. The total number of real parameters is $D+2 D K+1$. According to (18), the deterministic $C R B$ for $\overline{\boldsymbol{\theta}}$ can be expressed as [89, 90]

$$
\mathrm{CRB}_{\operatorname{det}}(\overline{\boldsymbol{\theta}})=\frac{p_{n}}{2 K}\left\{\operatorname{Re}\left[\left(\mathbf{U}_{\mathbb{S}}^{H} \boldsymbol{\Pi}_{\mathbf{V}_{\mathbb{S}}}^{\perp} \mathbf{U}_{\mathbb{S}}\right) \odot \widehat{\mathbf{P}}^{T}\right]\right\}^{-1}
$$


where

$$
\begin{aligned}
\mathbf{V}_{\mathbb{S}} & =\left[\begin{array}{llll}
\mathbf{v}_{\mathbb{S}}\left(\bar{\theta}_{1}\right) & \mathbf{v}_{\mathbb{S}}\left(\bar{\theta}_{2}\right) & \ldots & \mathbf{v}_{\mathbb{S}}\left(\bar{\theta}_{D}\right)
\end{array}\right] \\
\mathbf{U}_{\mathbb{S}} & =\left[\begin{array}{llll}
\frac{\partial \mathbf{v}_{\mathbb{S}}\left(\bar{\theta}_{1}\right)}{\partial \bar{\theta}_{1}} & \frac{\partial \mathbf{v}_{\mathbb{S}}\left(\bar{\theta}_{2}\right)}{\partial \bar{\theta}_{2}} & \ldots & \frac{\partial \mathbf{v}_{\mathbb{S}}\left(\bar{\theta}_{D}\right)}{\partial \bar{\theta}_{D}}
\end{array}\right], \\
\widehat{\mathbf{P}} & =\frac{1}{K} \sum_{k=1}^{K}\left[\begin{array}{c}
A_{1}(k) \\
A_{2}(k) \\
\vdots \\
A_{D}(k)
\end{array}\right]\left[\begin{array}{c}
A_{1}(k) \\
A_{2}(k) \\
\vdots \\
A_{D}(k)
\end{array}\right]
\end{aligned}
$$

and $\boldsymbol{\Pi}_{\mathbf{A}}^{\perp}$ is as defined in (3). Thus $\mathbf{V}_{\mathbb{S}}$ is the array manifold matrix, and $\widehat{\mathbf{P}}$ is the sample covariance of $\left[A_{1}, A_{2}, \ldots, A_{D}\right]^{T}$. Note that a nonsingular $\widehat{\mathbf{P}}$ is required for (20) being valid, which in turn requires that $K \geq D$.

The stochastic $C R B$ model [91] also uses $K$ snapshots as in (17). It is assumed that the noise is spatially and temporally uncorrelated, and, in addition, the source amplitudes are stochastic with mean zero and

$$
\mathbb{E}\left[\left[\begin{array}{c}
A_{1}\left(k_{1}\right) \\
A_{2}\left(k_{1}\right) \\
\vdots \\
A_{D}\left(k_{1}\right)
\end{array}\right]\left[\begin{array}{c}
A_{1}\left(k_{2}\right) \\
A_{2}\left(k_{2}\right) \\
\vdots \\
A_{D}\left(k_{2}\right)
\end{array}\right]^{H}\right]=\mathbf{P} \delta_{k_{1}, k_{2}}
$$

where $[\mathbf{P}]_{i, j}=\mathrm{E}\left[A_{i} A_{j}^{*}\right]$. Thus the probability model for stochastic CRB for $K$ snapshots is given by

$$
\left[\begin{array}{c}
\mathbf{x}_{\mathbb{S}}(1) \\
\mathbf{x}_{\mathbb{S}}(2) \\
\vdots \\
\mathbf{x}_{\mathbb{S}}(K)
\end{array}\right] \sim \mathcal{C N}\left(\mathbf{0},\left[\begin{array}{cccc}
\boldsymbol{\Sigma} & \mathbf{O} & \ldots & \mathbf{O} \\
\mathbf{O} & \boldsymbol{\Sigma} & \ldots & \mathbf{O} \\
\vdots & \vdots & \ddots & \vdots \\
\mathbf{O} & \mathbf{O} & \ldots & \boldsymbol{\Sigma}
\end{array}\right]\right)
$$

where

$$
\boldsymbol{\Sigma}=\mathbf{V}_{\mathbb{S}} \mathbf{P} \mathbf{V}_{\mathbb{S}}^{H}+p_{n} \mathbf{I}
$$

In this scenario, the parameter vector $\boldsymbol{\alpha}_{\text {sto }}$ is

$$
\boldsymbol{\alpha}_{\text {sto }}=\left[\bar{\theta}_{i}, \quad[\mathbf{P}]_{i, i}, \operatorname{Re}\left\{[\mathbf{P}]_{i, j}\right\}, \operatorname{Im}\left\{[\mathbf{P}]_{i, j}\right\}, p_{n}\right]^{T}
$$

where $1 \leq i \leq D$ and $i>j$. The number of real scalar parameters becomes $D+D^{2}+1$. This does not depend on $K$ (snapshots) unlike in the deterministic model. Using (25) and (27) yields the stochastic CRB expression for $\overline{\boldsymbol{\theta}}$ :

$$
\begin{aligned}
& \operatorname{CRB}_{\text {sto }}(\overline{\boldsymbol{\theta}})=\frac{p_{n}}{2 K}\left\{\operatorname { R e } \left[\left(\mathbf{U}_{\mathbb{S}}^{H} \mathbf{\Pi}_{\mathbf{V}_{\mathbb{S}}}^{\perp} \mathbf{U}_{\mathbb{S}}\right)\right.\right. \\
&\left.\left.\odot\left(\mathbf{P V}_{\mathbb{S}}^{H} \boldsymbol{\Sigma}^{-1} \mathbf{V}_{\mathbb{S}} \mathbf{P}\right)^{T}\right]\right\}^{-1},
\end{aligned}
$$

where $\mathbf{V}_{\mathbb{S}}, \mathbf{U}_{\mathbb{S}}, \mathbf{P}, \boldsymbol{\Sigma}$ are defined in (21), (22), (24), and (26), respectively. Note that the CRB expression (28) does not assume any prior information on the source covariance matrix $\mathbf{P}$, except that $\mathbf{P}$ is nonsingular for the inverse in (28) to exist.

In DOA estimation literature, it is often assumed that the sources are uncorrelated [7, 73, 84]. In such cases, a subtle distinction should be made depending upon whether we know apriori the fact that the sources are uncorrelated:
1. If the sources are uncorrelated but this information is not known a priori, then the CRB can be evaluated from the expression (28) with a diagonal $\mathbf{P}$.

2. Otherwise, if the sources are known a priori to be uncorrelated, then the off-diagonal entries of $\mathbf{P}$ are known to be zero. This prior information modifies the parameter vector $\boldsymbol{\alpha}$, the FIM $\mathcal{I}(\boldsymbol{\alpha})$, and the CRB expression. Hence, the CRB expression (28) cannot be applied. The closed-form CRB expression for this scenario was proposed in [40], which will be reviewed next.

The probability model for the stochastic model with uncorrelated sources and $K$ snapshots (17) is given by

$$
\left[\begin{array}{c}
\mathbf{x}_{\mathbb{S}}(1) \\
\mathbf{x}_{\mathbb{S}}(2) \\
\vdots \\
\mathbf{x}_{\mathbb{S}}(K)
\end{array}\right] \sim \mathcal{C N}\left(\mathbf{0},\left[\begin{array}{cccc}
\mathbf{R}_{\mathbb{S}} & \mathbf{O} & \ldots & \mathbf{O} \\
\mathbf{O} & \mathbf{R}_{\mathbb{S}} & \ldots & \mathbf{O} \\
\vdots & \vdots & \ddots & \vdots \\
\mathbf{O} & \mathbf{O} & \ldots & \mathbf{R}_{\mathbb{S}}
\end{array}\right]\right)
$$

where $\mathbf{R}_{\mathbb{S}}$ is defined in (7). The parameters to be estimated are composed of normalized DOAs $\bar{\theta}_{i}$, source powers $p_{i}$, and noise power $p_{n}$, so that the parameter vector $\boldsymbol{\alpha}_{\text {uncor }}$ becomes

$$
\boldsymbol{\alpha}_{\text {uncor }}=\left[\bar{\theta}_{i}, p_{i}, p_{n}\right]^{T},
$$

where $1 \leq i \leq D$. It can be seen that the number of real parameters is only $2 D+1$ in this case. According to [40], the CRB for $\overline{\boldsymbol{\theta}}$ can be expressed as

$$
\mathrm{CRB}_{\text {uncor }}(\overline{\boldsymbol{\theta}})=\frac{1}{K}\left(\mathbf{P D}^{H} \mathbf{G}\left(\mathbf{G}^{H} \widetilde{\mathbf{C}} \mathbf{G}\right)^{-1} \mathbf{G}^{H} \mathbf{D P}\right)^{-1}
$$

where

$$
\begin{aligned}
\widetilde{\mathbf{C}} & =\left(\mathbf{R}_{\mathbb{S}}^{T} \otimes \mathbf{R}_{\mathbb{S}}\right)+\frac{p_{n}^{2}}{|\mathbb{S}|-D} \operatorname{vec}\left(\boldsymbol{\Pi}_{\mathbf{V}_{\mathbb{S}}}\right) \operatorname{vec}^{H}\left(\boldsymbol{\Pi}_{\mathbf{V}_{\mathbb{S}}}\right), \\
\mathbf{D} & =\left(\mathbf{U}_{\mathbb{S}}^{*} \circ \mathbf{V}_{\mathbb{S}}\right)+\left(\mathbf{V}_{\mathbb{S}}^{*} \circ \mathbf{U}_{\mathbb{S}}\right),
\end{aligned}
$$

$\mathbf{G}$ is any matrix whose columns span the null space of $\left(\mathbf{V}_{\mathbb{S}}^{*} \circ \mathbf{V}_{\mathbb{S}}\right)^{H}$, and $\mathbf{P}=\operatorname{diag}\left(p_{1}, p_{2}, \ldots, p_{D}\right)$ is the source covariance matrix. $\mathbf{V}_{\mathbb{S}}$ and $\mathbf{U}_{\mathbb{S}}$ are given in (21) and (22), respectively. Eq. (31) will be called Jansson et al.'s CRB expression in this paper. However, if $D=|\mathbb{S}|$, then the denominator in (32) becomes zero, which makes (32) and Jansson et al.'s CRB expression (31) invalid. Furthermore, if $D>|\mathbb{S}|$, then $\boldsymbol{\Pi}_{\mathbf{V}_{\mathbb{S}}}$ is not well-defined.

Table 1 summarizes some CRB expressions along with their model assumptions, as in [90], [91], and [40]. The deterministic CRB expression [90] and the stochastic CRB expression [91] assume less sources than the number of sensors, namely $D<|\mathbb{S}|$, while Jansson et al.'s CRB expression $[40]$ implicitly assumes $D<|\mathbb{S}|$ so that the expression for $\boldsymbol{\Pi}_{\mathbf{V}_{\mathbb{S}}}$ is valid. For the complex amplitude $A_{i}$, the deterministic CRB expression [90] assumes unknown, nonrandom $A_{i}$, the stochastic CRB expression [91] supposes 
Table 1: Summary of several related CRB expressions for DOA estimation ${ }^{1}$

Deterministic or Conditional Stochastic or Unconditional [40] and the CRB expression $[90] \quad[91]$ proposed in this paper

\begin{tabular}{|c|c|c|c|}
\hline $\begin{array}{l}\text { Number of } \\
\text { sources, } D\end{array}$ & Known $(D<|\mathbb{S}|)$ & Known $(D<|\mathbb{S}|)$ & $\begin{array}{l}\text { Known }(D<|\mathbb{S}| \text { in }[40]) \\
(D<|\mathbb{S}| \text { or } D \geq|\mathbb{S}| \text { in the } \\
\text { proposed CRB expression })\end{array}$ \\
\hline $\begin{array}{c}\text { Normalized } \\
\text { DOAs, }\left\{\bar{\theta}_{i}\right\}_{i=1}^{D}\end{array}$ & Unknown, non-random & Unknown, non-random & Unknown, non-random \\
\hline $\begin{array}{l}\text { Complex } \\
\text { amplitude } \\
\left\{A_{i}\right\}_{i=1}^{D}\end{array}$ & Unknown, non-random & $\begin{array}{l}\text { Unknown, random, } \\
\text { correlated, } \\
{\left[A_{i}\right]_{i=1}^{D} \sim \mathcal{C N}(\mathbf{0}, \mathbf{P})}\end{array}$ & $\begin{array}{c}\text { Unknown, random, } \\
\text { uncorrelated, }\left[A_{i}\right]_{i=1}^{D} \sim \\
\mathcal{C N}\left(\mathbf{0}, \operatorname{diag}\left(p_{1}, \ldots, p_{D}\right)\right)\end{array}$ \\
\hline Noise vector $\mathbf{n}_{\mathbb{S}}$ & $\begin{array}{l}\text { Unknown, random, } \\
\mathbf{n}_{\mathbb{S}} \sim \mathcal{C} \mathcal{N}\left(\mathbf{0}, p_{n} \mathbf{I}\right)\end{array}$ & $\begin{array}{l}\text { Unknown, random, } \\
\mathbf{n}_{\mathbb{S}} \sim \mathcal{C N}\left(\mathbf{0}, p_{n} \mathbf{I}\right)\end{array}$ & $\begin{array}{l}\text { Unknown, random, } \\
\mathbf{n}_{\mathbb{S}} \sim \mathcal{C} \mathcal{N}\left(\mathbf{0}, p_{n} \mathbf{I}\right)\end{array}$ \\
\hline $\begin{array}{l}\text { Distribution of } \\
\mathbf{x}_{\mathbb{S}}\end{array}$ & $\mathcal{C N}\left(\sum_{i=1}^{D} A_{i} \mathbf{v}_{\mathbb{S}}\left(\bar{\theta}_{i}\right), p_{n} \mathbf{I}\right)$ & $\mathcal{C N}\left(\mathbf{0}, \mathbf{V}_{\mathbb{S}} \mathbf{P} \mathbf{V}_{\mathbb{S}}^{H}+p_{n} \mathbf{I}\right)$ & $\mathcal{C N}\left(\mathbf{0}, \mathbf{R}_{\mathbb{S}}\right)$ \\
\hline $\begin{array}{l}\text { Distribution of } \\
K \text { snapshots }\end{array}$ & $(18)$ & $(25)$ & $(29)$ \\
\hline $\begin{array}{l}\text { Unknown } \\
\text { parameters }\end{array}$ & $(19)$ & $(27)$ & $(30)$ \\
\hline $\begin{array}{l}\text { Number of } \\
\text { unknown } \\
\text { parameters }\end{array}$ & $D+2 D K+1$ & $D+D^{2}+1$ & $2 D+1$ \\
\hline
\end{tabular}

$\operatorname{CRB}(\overline{\boldsymbol{\theta}})$

$(31)$, or

the new expression proposed in Theorem 2

\footnotetext{
${ }^{1} \overline{\boldsymbol{\theta}}=\left[\bar{\theta}_{1}, \ldots, \bar{\theta}_{D}\right]^{T},[\mathbf{P}]_{i, j}=\mathbb{E}\left[A_{i} A_{j}^{*}\right], \mathbf{V}_{\mathbb{S}}=\left[\mathbf{v}_{\mathbb{S}}\left(\bar{\theta}_{1}\right), \ldots, \mathbf{v}_{\mathbb{S}}\left(\bar{\theta}_{D}\right)\right], \mathbf{R}_{\mathbb{S}}=\sum_{i=1}^{D} p_{i} \mathbf{v}_{\mathbb{S}}\left(\bar{\theta}_{i}\right) \mathbf{v}_{\mathbb{S}}^{H}\left(\bar{\theta}_{i}\right)+p_{n} \mathbf{I}$
} 
unknown, random $A_{i}$ with mean zero and covariance $\mathbf{P}$, while Jansson et al.'s CRB expression presumes unknown, random $A_{i}$ with mean zero and a diagonal covariance matrix.

The CRB for DOA estimation with sparse arrays is gaining importance due to recent developments in coarraybased DOA estimators and sparse array design. An early work by Abramovich et al. [1] demonstrated numerically that the CRB exhibits two different behaviors in the regimes $D<|\mathbb{S}|$ and $D \geq|\mathbb{S}|$. For $D<|\mathbb{S}|$, the CRB at high SNR decays to zero. For $D \geq|\mathbb{S}|$, the CRB at high SNR tends to saturate to a non-zero value as SNR increases. Among other results, we will prove both of these in this paper (Theorems 3 and 4 ).

The connection between the CRB and the array geometry has been studied in previous literature. The CRB for single source with azimuth, elevation, and planar arrays was investigated in [16]. It was observed that the CRB for DOAs is inversely proportional to the array variance, under suitable assumptions. The CRB for two sources with one-dimensional DOA profiles was considered in [99, 100], based on the deterministic CRB expression, (20). It was noted empirically that larger array aperture helps to reduce the CRB and the array geometry influences the maximum number of identifiable sources.

Another interesting work by Koochakzadeh and Pal formulates the DOA estimation using a predefined DOA grid and sensor perturbation [44, 45]. The DOA grid is denoted by $\vartheta_{1}, \vartheta_{2}, \ldots, \vartheta_{N_{\vartheta}}$ while the perturbation is characterized by $\delta \in \mathbb{R}^{|\mathbb{S}|}$. In particular, the measurement $\mathbf{y}(k)$ originates from the following data model:

$$
\mathbf{y}(k)=\mathbf{A}_{\text {grid }} \mathbf{x}(k)+\mathbf{w}(k) \quad \in \mathbb{C}^{|\mathbb{S}|}, \quad k=1,2, \ldots, K,
$$

where $\mathbf{x}(k) \in \mathbb{C}^{N_{\vartheta}}$ is the source amplitude on the grid with source powers $\gamma_{i}=\mathbb{E}\left[\left|[\mathbf{x}(k)]_{i}\right|^{2}\right] . \mathbf{w}(k)$ is the noise term. $\mathbf{A}_{\text {grid }} \in \mathbb{C}^{|\mathbb{S}| \times N_{\vartheta}}$ is given by $\left[\mathbf{v}_{\mathbb{S}}\left(\bar{\vartheta}_{1}\right), \mathbf{v}_{\mathbb{S}}\left(\bar{\vartheta}_{2}\right), \ldots, \mathbf{v}_{\mathbb{S}}\left(\bar{\vartheta}_{N_{\vartheta}}\right)\right]$. In this setting, the parameters to be estimated are the source powers $\gamma_{i}$ and the sensor location perturbation $\boldsymbol{\delta}$. The FIM and the CRB for $\gamma_{i}$ were also analyzed in detail.

\section{New Expressions for CRB, Applicable for Sparse Arrays with More Sources than Sensors}

\subsection{Remarks on the CRB expressions [40, 90, 91]}

We now argue that, among the three CRB expressions: the deterministic CRB expression [90], the stochastic CRB expression [91], and Jansson et al.'s CRB expression [40], in Section 3, only Jansson et al.'s CRB expression is appropriate when coarray-based methods are applicable for DOA estimation.

The deterministic CRB expression [90] is not suitable for coarray-based DOA estimators since the assumptions do not match. It is assumed in the deterministic model that the complex amplitude $A_{i}$ is deterministic, as summarized in Table 1. Coarray-based DOA estimators operate under the condition that sources are stochastic, as mentioned in (5).

The stochastic CRB expression in [91] is incompatible with coarray-based DOA estimators due to the following:

1. The stochastic CRB expression in [91] is valid if $D<|\mathbb{S}|$. Hence, it is inappropriate to consider the stochastic CRB expression for the regime $D \geq|\mathbb{S}|$ where coarray based DOA estimators are usually of interest.

2. The assumptions are different. The stochastic CRB expression in [91] considers the stochastic model with source covariance $\mathbf{P}$. But it is not known apriori that the sources are uncorrelated. On the other hand, for coarray-based DOA estimators, it is known a priori that sources are uncorrelated, as stated in (5).

Finally, Jansson et al.'s CRB expression [40] is applicable to coarray-based DOA estimators. It is because the uncorrelated information is assumed to be known a priori, which matches the assumptions of coarray-based DOA estimators.

To demonstrate how much the uncorrelated prior helps to reduce the CRB, consider a sensor array with $\mathbb{S}=$ $\{0,1,4,6\}$. Assume there are two equal-power, uncorre-

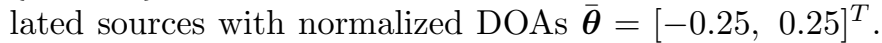
The number of snapshots $K$ is 500 and the SNR is $0 \mathrm{~dB}$. Substituting these parameters into (28) and (31) yields

The stochastic CRB expression [91] :

$$
\begin{aligned}
& \quad[\operatorname{CRB}(\overline{\boldsymbol{\theta}})]_{1,1}=[\operatorname{CRB}(\overline{\boldsymbol{\theta}})]_{2,2}=1.809 \times 10^{-6}, \\
& \text { Jansson et al.'s CRB expression }[40]: \\
& \quad[\mathrm{CRB}(\overline{\boldsymbol{\theta}})]_{1,1}=[\operatorname{CRB}(\overline{\boldsymbol{\theta}})]_{2,2}=1.696 \times 10^{-6} .
\end{aligned}
$$

Thus, Jansson et al.'s CRB (with uncorrelated prior) is less than the stochastic CRB (without uncorrelated prior).

However, Jansson et al.'s CRB expression has some limitations. First of all, the precise conditions that Jansson et al.'s CRB expression is valid are not explicitly stated in [40]. From Jansson et al.'s CRB expression, it is not so easy to study the behavior of the CRB with respect to the number of snapshots $K$, the number of sources $D$, and the SNR. Furthermore, [40] considers only the ULA and it is not clear from [40] how sparse arrays, like MRA, nested arrays, and coprime arrays, influence the CRB. Finally, for $D=|\mathbb{S}|$, Jansson et al.'s CRB expression becomes undefined, due to the appearance of $|\mathbb{S}|-D$ in the denominator of $(32)$.

In Section 4.2, we will propose a CRB expression that addresses all these issues raised in the previous paragraph. First, it will be shown that a rank condition on the augmented coarray manifold (ACM) matrix is necessary and sufficient for the nonsingular FIM, which leads to a closedfrom CRB expression.

\subsection{The proposed Cramér-Rao bound expression}

Consider a random vector $\mathbf{x}$ with a complex normal distribution with mean zero and covariance $\boldsymbol{\Sigma}(\boldsymbol{\alpha})$, where $\boldsymbol{\alpha}$ is 
a real-valued parameter vector. The $(p, \ell)$ th entry of the FIM $\mathcal{I}(\boldsymbol{\alpha})$ is given by $[88,89,91]$

$$
[\mathcal{I}(\boldsymbol{\alpha})]_{p, \ell}=\operatorname{tr}\left(\boldsymbol{\Sigma}^{-1}(\boldsymbol{\alpha}) \frac{\partial \boldsymbol{\Sigma}(\boldsymbol{\alpha})}{\partial[\boldsymbol{\alpha}]_{p}} \boldsymbol{\Sigma}^{-1}(\boldsymbol{\alpha}) \frac{\partial \boldsymbol{\Sigma}(\boldsymbol{\alpha})}{\partial[\boldsymbol{\alpha}]_{\ell}}\right) .
$$

Setting the probability model to be (29) and the parameter vector to be (30) results in

$$
\begin{aligned}
& {[\mathcal{I}(\boldsymbol{\alpha})]_{p, \ell}=K \operatorname{tr}\left(\mathbf{R}_{\mathbb{S}}^{-1} \frac{\partial \mathbf{R}_{\mathbb{S}}}{\partial[\boldsymbol{\alpha}]_{p}} \mathbf{R}_{\mathbb{S}}^{-1} \frac{\partial \mathbf{R}_{\mathbb{S}}}{\partial[\boldsymbol{\alpha}]_{\ell}}\right)} \\
& =K\left[\operatorname{vec}\left(\frac{\partial \mathbf{R}_{\mathbb{S}}}{\partial[\boldsymbol{\alpha}]_{p}}\right)\right]^{H}\left(\mathbf{R}_{\mathbb{S}}^{-T} \otimes \mathbf{R}_{\mathbb{S}}^{-1}\right) \operatorname{vec}\left(\frac{\partial \mathbf{R}_{\mathbb{S}}}{\partial[\boldsymbol{\alpha}]_{\ell}}\right) \\
& =K\left[\left(\mathbf{R}_{\mathbb{S}}^{T} \otimes \mathbf{R}_{\mathbb{S}}\right)^{-\frac{1}{2}} \frac{\partial \mathbf{r}_{\mathbb{S}}}{\partial[\boldsymbol{\alpha}]_{p}}\right]^{H}\left[\left(\mathbf{R}_{\mathbb{S}}^{T} \otimes \mathbf{R}_{\mathbb{S}}\right)^{-\frac{1}{2}} \frac{\partial \mathbf{r}_{\mathbb{S}}}{\partial[\boldsymbol{\alpha}]_{\ell}}\right],
\end{aligned}
$$

since $\operatorname{tr}(\mathbf{A B C D})=\operatorname{vec}\left(\mathbf{B}^{H}\right)^{H}\left(\mathbf{A}^{T} \otimes \mathbf{C}\right) \operatorname{vec}(\mathbf{D})$, and $(\mathbf{A} \otimes$ $\mathbf{B})^{-1}=\mathbf{A}^{-1} \otimes \mathbf{B}^{-1}$ for nonsingular $\mathbf{A}$ and $\mathbf{B}[56]$. The vector $\mathbf{r}_{\mathbb{S}}$ is defined as

$$
\mathbf{r}_{\mathbb{S}}=\operatorname{vec}\left(\mathbf{R}_{\mathbb{S}}\right)
$$

Eq. (37) leads to an expression for the FIM $\mathcal{I}(\boldsymbol{\alpha})$ as follows

$$
\begin{aligned}
& \mathcal{I}(\boldsymbol{\alpha})=K\left[\begin{array}{l}
\mathbf{G}^{H} \\
\boldsymbol{\Delta}^{H}
\end{array}\right]\left[\begin{array}{ll}
\mathbf{G} & \boldsymbol{\Delta}
\end{array}\right] \\
& =\quad D \downarrow\left[\begin{array}{lc}
\stackrel{D}{K \mathbf{G}^{H} \mathbf{G}} & \stackrel{D+1}{K \mathbf{G}^{H} \boldsymbol{\Delta}} \\
K \boldsymbol{\Delta}^{H} \mathbf{G} & K \boldsymbol{\Delta}^{H} \boldsymbol{\Delta}
\end{array}\right],
\end{aligned}
$$

where

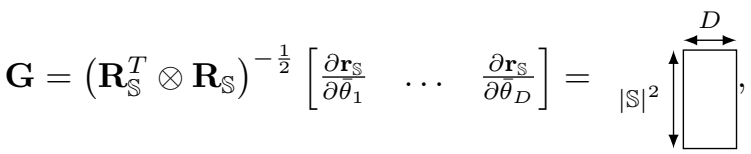

$$
\begin{aligned}
& \boldsymbol{\Delta}=\left(\mathbf{R}_{\mathbb{S}}^{T} \otimes \mathbf{R}_{\mathbb{S}}\right)^{-\frac{1}{2}}\left[\begin{array}{llll}
\frac{\partial \mathbf{r}_{\mathbb{S}}}{\partial p_{1}} & \cdots & \frac{\partial \mathbf{r}_{\mathbb{S}}}{\partial p_{D}} & \frac{\partial \mathbf{r}_{\mathbb{S}}}{\partial p_{n}}
\end{array}\right]=\underset{|\mathbb{S}|^{2}}{\stackrel{D+1}{\rightleftarrows} .}
\end{aligned}
$$

It follows from (39) that the FIM is positive semidefinite. And $\boldsymbol{\Delta}^{H} \boldsymbol{\Delta}$ is obviously positive semidefinite. If the FIM $\boldsymbol{I}(\boldsymbol{\alpha})$ is nonsingular, then the CRB for the normalized DOAs $\overline{\boldsymbol{\theta}}=\left[\bar{\theta}_{1}, \ldots, \bar{\theta}_{D}\right]^{T}$ can be expressed as the inverse of the Schur complement of the block $\boldsymbol{\Delta}^{H} \boldsymbol{\Delta}$ of the FIM $\mathcal{I}(\boldsymbol{\alpha})[88]$

$$
\operatorname{CRB}(\overline{\boldsymbol{\theta}})=\frac{1}{K}\left(\mathbf{G}^{H} \boldsymbol{\Pi}_{\Delta}^{\perp} \mathbf{G}\right)^{-1}
$$

where $\boldsymbol{\Pi}_{\boldsymbol{\Delta}}^{\perp}=\mathbf{I}-\boldsymbol{\Delta}\left(\boldsymbol{\Delta}^{H} \boldsymbol{\Delta}\right)^{-1} \boldsymbol{\Delta}^{H}$ is defined as in (3).

An important observation here is that nonsingularity of the FIM is equivalent to nonsingularity of $\boldsymbol{\Delta}^{H} \boldsymbol{\Delta}$ and $\mathbf{G}^{H} \mathbf{\Pi}_{\Delta}^{\perp} \mathbf{G}$ :
Lemma 1. Let $\mathbf{F}$ be a positive semidefinite matrix of the form

$$
\mathbf{F}=\left[\begin{array}{cc}
\mathbf{A} & \mathbf{B} \\
\mathbf{B}^{H} & \mathbf{D}
\end{array}\right] \succeq \mathbf{0},
$$

where $\mathbf{A}$ and $\mathbf{D}$ are Hermitian matrices. Then $\mathbf{F}$ is nonsingular (invertible) if and only if $\mathbf{D}$ and the Schur complement of $\mathbf{D}$, namely, $\mathbf{A}-\mathbf{B D}^{-1} \mathbf{B}^{H}$, are both nonsingular.

Proof. The proof can be found in Appendix A.2.

Lemma 1 can be applied to (40). Let $\mathbf{F}$ be the FIM $\mathcal{I}(\boldsymbol{\alpha})$, which is positive semidefinite. The submatrices $\mathbf{A}=K \mathbf{G}^{H} \mathbf{G}, \mathbf{B}=K \mathbf{G}^{H} \boldsymbol{\Delta}$, and $\mathbf{D}=K \boldsymbol{\Delta}^{H} \boldsymbol{\Delta}$ so that the Schur complement of $\mathbf{D}$ becomes $\mathbf{A}-\mathbf{B D}^{-1} \mathbf{B}^{H}=$ $K \mathbf{G}^{H} \mathbf{\Pi}_{\Delta}^{\perp} \mathbf{G}$. Lemma 1 indicates that the FIM $\mathcal{I}(\boldsymbol{\alpha})$ is nonsingular if and only if $\boldsymbol{\Delta}^{H} \boldsymbol{\Delta}$ and $\mathbf{G}^{H} \boldsymbol{\Pi}_{\boldsymbol{\Delta}}^{\perp} \mathbf{G}$ are both nonsingular.

It is of great interest to simplify the condition that $\boldsymbol{\Delta}^{H} \boldsymbol{\Delta}$ and $\mathbf{G}^{H} \boldsymbol{\Pi}_{\boldsymbol{\Delta}}^{\perp} \mathbf{G}$ are both nonsingular. The following lemmas characterize the necessary and sufficient conditions that $\boldsymbol{\Delta}^{H} \boldsymbol{\Delta}$ and $\mathbf{G}^{H} \boldsymbol{\Pi}_{\boldsymbol{\Delta}}^{\perp} \mathbf{G}$ are positive definite, hence nonsingular. For the following lemma, the reader should recall the triangular bracket notation from Section 1.1.

Lemma 2. Let $\mathbf{V}_{\mathbb{D}}$ be the array manifold matrix defined on the difference coarray and $\mathbf{W}_{\mathbb{D}}=\left[\mathbf{V}_{\mathbb{D}}, \mathbf{e}_{0}\right]$, where $\mathbf{e}_{0}$ is a column vector satisfying $\left\langle\mathbf{e}_{0}\right\rangle_{m}=\delta_{m, 0}$ for $m \in \mathbb{D}$. Therefore, $\mathbf{V}_{\mathbb{D}}$ is a $|\mathbb{D}| \times D$ matrix while $\mathbf{W}_{\mathbb{D}}$ has size $|\mathbb{D}| \times$ $(D+1)$. Then $\boldsymbol{\Delta}^{H} \boldsymbol{\Delta}$ is positive definite if and only if

$$
\operatorname{rank}\left(\mathbf{W}_{\mathbb{D}}\right)=D+1,
$$

i.e., if and only if $\mathbf{W}_{\mathbb{D}}$ has full column rank.

Proof. The proof can be found in Appendix A.3.

Definition 4 (ACM matrix). The augmented coarray manifold (ACM) matrix is defined as

$$
\begin{aligned}
& \mathbf{A}_{c}=\left[\operatorname{diag}(\mathbb{D}) \mathbf{V}_{\mathbb{D}} \quad \mathbf{W}_{\mathbb{D}}\right]
\end{aligned}
$$

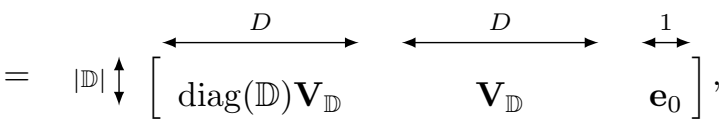

where $\operatorname{diag}(\mathbb{D})$ is a diagonal matrix with $\mathbb{D}$ on its diagonals, $\mathbf{V}_{\mathbb{D}}$ is the array manifold matrix defined on $\mathbb{D}$, and $\mathbf{W}_{\mathbb{D}}=$ $\left[\mathbf{V}_{\mathbb{D}}, \mathbf{e}_{0}\right]$, where $\mathbf{e}_{0}$ is a column vector satisfying $\left\langle\mathbf{e}_{0}\right\rangle_{m}=$ $\delta_{m, 0}$ for $m \in \mathbb{D}$. The triangular brackets $\langle\cdot\rangle$ are defined in Section 1.1.

Lemma 3. Assume that $\operatorname{rank}\left(\mathbf{W}_{\mathbb{D}}\right)=D+1$ and let $\mathbf{A}_{c}$ be the augmented coarray manifold (ACM) matrix. Then $\mathbf{G}^{H} \mathbf{\Pi}_{\Delta}^{\perp} \mathbf{G}$ is positive definite if and only if

$$
\operatorname{rank}\left(\mathbf{A}_{c}\right)=2 D+1,
$$

i.e., if and only if the ACM matrix $\mathbf{A}_{c}$ has full column rank. 
Proof. The proof can be found in Appendix A.4.

The significance of Lemma 2 and Lemma 3 is that the invertibility of $\boldsymbol{\Delta}^{H} \boldsymbol{\Delta}$ and $\mathbf{G}^{H} \boldsymbol{\Pi}_{\boldsymbol{\Delta}}^{\perp} \mathbf{G}$ can be simply characterized by (44) and (47). Furthermore, these conditions lead to a necessary and sufficient condition for nonsingular FIMs, as summarized next:

Theorem 1. Let $\mathbf{A}_{c}$ be the ACM matrix, as defined in Definition 4. Then the FIM $\mathcal{I}(\boldsymbol{\alpha})$, given in (36), is nonsingular if and only if $\mathbf{A}_{c}$ has full column rank, i.e., if and only if

$$
\operatorname{rank}\left(\mathbf{A}_{c}\right)=2 D+1
$$

Proof. It follows directly from Lemma 1, 2, and 3.

The next result is that, if the FIM is nonsingular, then the CRB exists and the closed-form CRB expression is given by the following theorem. The quantity $\mathbf{J}$ in this theorem is defined in Definition 5 in Appendix B.

Theorem 2. Let $\mathbf{A}_{c}$ be the ACM matrix, as defined in Definition 4. If $\operatorname{rank}\left(\mathbf{A}_{c}\right)=2 D+1$, then the CRB for normalized DOAs $\overline{\boldsymbol{\theta}}=\left[\bar{\theta}_{1}, \ldots, \bar{\theta}_{D}\right]^{T}$ can be expressed as

$$
\operatorname{CRB}(\overline{\boldsymbol{\theta}})=\frac{1}{4 \pi^{2} K}\left(\mathbf{G}_{0}^{H} \boldsymbol{\Pi}_{\mathbf{M} \mathbf{W}_{\mathbb{D}}}^{\perp} \mathbf{G}_{0}\right)^{-1}
$$

where

$$
\begin{aligned}
& \mathbf{G}_{0}=\mathbf{M}(\operatorname{diag}(\mathbb{D})) \times \mathbf{V}_{\mathbb{D}} \times\left(\operatorname{diag}\left(p_{1}, p_{2}, \ldots, p_{D}\right)\right), \\
& \mathbf{M}=\left(\mathbf{J}^{H}\left(\mathbf{R}_{\mathbb{S}}^{T} \otimes \mathbf{R}_{\mathbb{S}}\right)^{-1} \mathbf{J}\right)^{\frac{1}{2}}, \\
& \mathbf{R}_{\mathbb{S}}=\sum_{i=1}^{D} p_{i} \mathbf{v}_{\mathbb{S}}\left(\bar{\theta}_{i}\right) \mathbf{v}_{\mathbb{S}}^{H}\left(\bar{\theta}_{i}\right)+p_{n} \mathbf{I} \\
& \mathbf{V}_{\mathbb{D}}=\left[\begin{array}{llll}
\mathbf{v}_{\mathbb{D}}\left(\bar{\theta}_{1}\right) & \mathbf{v}_{\mathbb{D}}\left(\bar{\theta}_{2}\right) & \ldots & \mathbf{v}_{\mathbb{D}}\left(\bar{\theta}_{D}\right)
\end{array}\right], \\
& \mathbf{W}_{\mathbb{D}}=\left[\begin{array}{ll}
\mathbf{V}_{\mathbb{D}} & \mathbf{e}_{0}
\end{array}\right] \text {. }
\end{aligned}
$$

Here $K$ is the number of snapshots, $D$ is the number of sources, $p_{i}$ is the ith source power, and $p_{n}$ is the noise power.

Recall that, $\mathbb{D}$ is the difference coarray, as defined in Definition 1, and $\mathbf{J}$ is given in Definition 5 of Appendix B. $\mathbf{V}_{\mathbb{D}}$ is the array manifold matrix on $\mathbb{D}$. $\mathbf{e}_{0}$ is defined in Lemma 2. The matrix $\boldsymbol{\Pi}_{\mathbf{A}}^{\perp}=\mathbf{I}-\mathbf{A}\left(\mathbf{A}^{H} \mathbf{A}\right)^{-1} \mathbf{A}^{H}$ is defined in (3).

Proof. The proof of this Theorem follows from Appendix A.1, Appendix A.4, (A.11), and (43).

\subsection{Comparison between [1, 40] and the proposed CRB expression}

In this subsection, we will include more detailed discussions on the CRB expressions [1, 40] and the proposed CRB expression (Theorem 2). These expressions are equivalent under appropriate assumptions.
Abramovich et al. [1] plotted the CRB curves numerically based on the FIM of the complex normal distribution (36) (or (11) in [1]). It is also known a priori that sources are uncorrelated and there is no assumption on the number of sources. As a result, their CRB plots should be identical to those from Theorem 2, for any choice of parameters. However, Abramovich et al.'s CRB expressions make it difficult to explain the number of resolvable sources, the behavior of the CRB for large SNR, and the conditions under which the FIM is nonsingular.

Jansson et al.'s CRB expressions [40] were derived from (43) (or (38) in [40]). Then, to simplify (43) into (31), the projection matrix $\Pi_{\mathbf{V}_{\mathbb{S}}}=\mathbf{V}_{\mathbb{S}}\left(\mathbf{V}_{\mathbb{S}}^{H} \mathbf{V}_{\mathbb{S}}\right)^{-1} \mathbf{V}_{\mathbb{S}}^{H}$ was introduced. Note that, if $D>|\mathbb{S}|$, then $\mathbf{V}_{\mathbb{S}}^{H} \mathbf{V}_{\mathbb{S}}$ is singular, so $\boldsymbol{\Pi}_{\mathbf{V}_{\mathbb{S}}}$ and (31) are undefined. However, for certain parameters, if (31) is well-defined, then it should agree with (49) in Theorem 2, since they are both derived from (43).

The proposed CRB expressions overcome the limitations of $[1,40]$, as we shall see in Section 5 and 6 . Later on, all these CRB expressions will be compared through numerical examples in Section 7.4 and Fig. 7.

\section{Conclusions which Follow from Theorem 2}

Theorem 2 enables us to study various parameters that affect the CRB, such as the array configuration, the normalized DOAs, the number of snapshots, and the SNR, as explained next.

Property 3. The rank condition, (48), depends only on four factors: the difference coarray $\mathbb{D}$, the normalized DOAs $\overline{\boldsymbol{\theta}}$, the number of sources $D$, and $\mathbf{e}_{0}$. The following parameters are irrelevant to (48): The source powers $p_{1}, \ldots, p_{D}$, the noise power $p_{n}$, and the number of snapshots $K$.

Property 4. The CRB for $\overline{\boldsymbol{\theta}}$ is a function of the physical array $\mathbb{S}$, the normalized DOA $\overline{\boldsymbol{\theta}}$, the number of sources $D$, the number of snapshots $K$, and the SNR of sources $p_{1} / p_{n}, \ldots, p_{D} / p_{n}$.

The fact that the CRB depends on the SNRs and not on individual powers can be proved as follows: If we replace $p_{i}$ and $p_{n}$ with $C p_{i}$ and $C p_{n}$, then $\mathbf{R}_{\mathbb{S}}, \mathbf{M}$, and $\mathbf{G}_{0}$ change to

$$
\begin{aligned}
& \mathbf{R}_{\mathbb{S}}^{\prime}=\sum_{i=1}^{D} C p_{i} \mathbf{v}_{\mathbb{S}}\left(\bar{\theta}_{i}\right) \mathbf{v}_{\mathbb{S}}^{H}\left(\bar{\theta}_{i}\right)+C p_{n} \mathbf{I}=C \mathbf{R}_{\mathbb{S}}, \\
& \mathbf{M}^{\prime}=\left(\mathbf{J}^{H}\left(\left(C \mathbf{R}_{\mathbb{S}}\right)^{T} \otimes\left(C \mathbf{R}_{\mathbb{S}}\right)\right)^{-1} \mathbf{J}\right)^{\frac{1}{2}}=C^{-1} \mathbf{M} \\
& \mathbf{G}_{0}^{\prime}=\mathbf{M}^{\prime}(\operatorname{diag}(\mathbb{D})) \mathbf{V}_{\mathbb{D}}\left(\operatorname{diag}\left(C p_{1}, C p_{2}, \ldots, C p_{D}\right)\right)=\mathbf{G}_{0} .
\end{aligned}
$$

Therefore, $\mathbf{G}_{0}^{\prime H} \mathbf{\Pi}_{\mathbf{M}^{\prime} \mathbf{W}_{\mathbb{D}}}^{\perp} \mathbf{G}_{0}^{\prime}=\mathbf{G}_{0}^{H} \boldsymbol{\Pi}_{\mathbf{M} \mathbf{W}_{\mathbb{D}}}^{\perp} \mathbf{G}_{0}$, implying that the $\mathrm{CRB}$ is unchanged if all the ratios $p_{i} / p_{n}$ are unchanged.

Property 3 characterizes the parameters that affect the singularity of the FIM, due to Theorem 1. If two distinct array configurations $\mathbb{S}_{1}$ and $\mathbb{S}_{2}$ have the same difference 
coarray $\mathbb{D}$, then for the same DOAs, the ACM matrices are exactly identical.

Example 1. For instance, consider the nested array with $N_{1}=N_{2}=5$ and the second-order super nested array with $N_{1}=N_{2}=5$. The sensor locations are given by $[51,63]$ :

$$
\begin{aligned}
\mathbb{S}_{\text {nested }} & =\{1,2,3,4,5,6,12,18,24,30\}, \\
\mathbb{S}_{\text {super nested }} & =\{1,3,5,8,10,12,18,24,29,30\} .
\end{aligned}
$$

It was proved in [51] that their difference coarray are identical, i.e., $\mathbb{D}_{\text {nested }}=\mathbb{D}_{\text {super nested }}=\{-29, \ldots, 29\}$. Hence,

$$
\left(\mathbf{A}_{c}\right)_{\text {nested }}=\left(\mathbf{A}_{c}\right)_{\text {super nested }}
$$

The above equation indicates that for some normalized DOAs $\overline{\boldsymbol{\theta}}^{\star}$, if the nested array (55) leads to a singular FIM, then the super nested array (56) also results in a singular FIM for the same $\overline{\boldsymbol{\theta}}^{\star}$.

However, two distinct array configurations $\mathbb{S}_{1}$ and $\mathbb{S}_{2}$ with the same difference coarray do not necessarily imply the same CRB. This is because, as in (51), the matrix $\mathbf{M}=\left(\mathbf{J}^{H}\left(\mathbf{R}_{\mathbb{S}}^{T} \otimes \mathbf{R}_{\mathbb{S}}\right)^{-1} \mathbf{J}\right)^{\frac{1}{2}}$ depends on $\mathbb{S}$.

Example 2. To demonstrate, consider the nested array and the super nested array in (55) and (56). Let $\overline{\boldsymbol{\theta}}=$ $[0.1,0.2]^{T}, p_{1}=p_{2}=p_{n}=1$, and $K=500$. Evaluating (49) yields

$$
\begin{aligned}
& \text { Nested array: } \\
& \qquad[\mathrm{CRB}(\overline{\boldsymbol{\theta}})]_{1,1}=[\mathrm{CRB}(\overline{\boldsymbol{\theta}})]_{2,2}=3.2648 \times 10^{-8},
\end{aligned}
$$

Super nested array:

$$
[\mathrm{CRB}(\overline{\boldsymbol{\theta}})]_{1,1}=[\mathrm{CRB}(\overline{\boldsymbol{\theta}})]_{2,2}=2.9352 \times 10^{-8} .
$$

Therefore, the CRBs are indeed different even if the difference coarrays are identical.

Property 5. If $\operatorname{rank}\left(\mathbf{A}_{c}\right)=2 D+1$, then as the number of snapshots $K$ approaches infinity, $\operatorname{CRB}(\overline{\boldsymbol{\theta}})$ converges to zero.

Proof. This follows directly from the expression (49).

The following theorems investigate the asymptotic behavior of the CRB for large SNR. Assume the sources have identical power. It was experimentally noticed in [1] that for $D<|\mathbb{S}|$, the CRB decays to zero for large SNR while for $D \geq|\mathbb{S}|$, the CRB tends to converge to a non-zero value for large SNR. Here we find these phenomena to be a provable consequence of the proposed CRB expression as given in Theorem 2 .

However, in this paper, we notice that the conditions $D<|\mathbb{S}|$ and $D \geq|\mathbb{S}|$ are not fundamental to the asymptotic behavior of the CRB for large SNR. Instead, the condition that the array manifold matrix $\mathbf{V}_{\mathbb{S}}$ has full row rank, i.e., $\operatorname{rank}\left(\mathbf{V}_{\mathbb{S}}\right)=|\mathbb{S}|$, is more critical. In the regime $D<|\mathbb{S}|, \mathbf{V}_{\mathbb{S}}$ does not have full row rank since $\mathbf{V}_{\mathbb{S}}$ is a tall matrix. Thus, the asymptotic CRB expression can be specified by the following theorem:
Theorem 3. If the $D$ uncorrelated sources have equal SNR $p / p_{n}, \operatorname{rank}\left(\mathbf{V}_{\mathbb{S}}\right)<|\mathbb{S}|$, and $\operatorname{rank}\left(\mathbf{A}_{c}\right)=2 D+1$, then for sufficiently large $S N R$, the $C R B$ has the following asymptotic expression which converges to zero as $S N R$ tends to infinity:

$$
\operatorname{CRB}(\overline{\boldsymbol{\theta}}) \mid \underset{\operatorname{rank}\left(\mathbf{V}_{\mathbb{S}}\right)<|\mathbb{S}|}{\operatorname{largeSR}}=\frac{p_{n}}{4 \pi^{2} K p} \mathbf{S}^{-1},
$$

where

$$
\begin{aligned}
\mathbf{S}= & \mathbf{G}_{\infty}^{H} \boldsymbol{\Pi}_{\mathbf{M}_{\infty} \mathbf{W}_{\mathbb{D}}} \mathbf{G}_{\infty}+\frac{\left(\mathbf{G}_{\infty}^{H} \mathbf{u}\right)\left(\mathbf{G}_{\infty}^{H} \mathbf{u}\right)^{H}}{\|\mathbf{u}\|^{2}}, \\
\mathbf{M}_{\infty}= & {\left[\mathbf { J } ^ { H } \left[\left(\mathbf{U}_{s} \boldsymbol{\Lambda}^{-1} \mathbf{U}_{s}^{H}\right)^{T} \otimes\left(\mathbf{U}_{n} \mathbf{U}_{n}^{H}\right)\right.\right.} \\
& \left.\left.+\left(\mathbf{U}_{n} \mathbf{U}_{n}^{H}\right)^{T} \otimes\left(\mathbf{U}_{s} \boldsymbol{\Lambda}^{-1} \mathbf{U}_{s}^{H}\right)\right] \mathbf{J}\right]^{\frac{1}{2}}, \\
\mathbf{G}_{\infty}= & \mathbf{M}_{\infty}(\operatorname{diag}(\mathbb{D})) \mathbf{V}_{\mathbb{D}}, \\
\mathbf{u}= & \left(\mathbf{M}_{\infty} \mathbf{W}_{\mathbb{D}}\right)\left(\mathbf{W}_{\mathbb{D}}^{H} \mathbf{M}_{\infty}^{2} \mathbf{W}_{\mathbb{D}}\right)^{-1} \mathbf{e}_{D+1}, \\
\mathbf{e}_{D+1}= & {[\overbrace{0, \ldots, 0}^{D}, 1]^{T} . }
\end{aligned}
$$

$\mathbf{W}_{\mathbb{D}}^{H} \mathbf{M}_{\infty}^{2} \mathbf{W}_{\mathbb{D}}$ and $\mathbf{S}$ are assumed to be nonsingular. the vector $\mathbf{u}$ is assumed to be non-zero.

Here $\mathbf{V}_{\mathbb{S}} \mathbf{V}_{\mathbb{S}}^{H}$ has eigen-decomposition $\mathbf{U}_{s} \boldsymbol{\Lambda} \mathbf{U}_{s}^{H}$. $\mathbf{U}_{s}$ has dimension $|\mathbb{S}| \times \operatorname{rank}\left(\mathbf{V}_{\mathbb{S}}\right)$ with normalized eigenvectors on its columns. $\boldsymbol{\Lambda}$ is a $\operatorname{rank}\left(\mathbf{V}_{\mathbb{S}}\right) \times \operatorname{rank}\left(\mathbf{V}_{\mathbb{S}}\right)$ diagonal matrix with eigenvalues on its diagonals. The eigendecomposition of $\mathbf{R}_{\mathbb{S}}$ is $\mathbf{U}_{s}\left(p \boldsymbol{\Lambda}+p_{n} \mathbf{I}\right) \mathbf{U}_{s}^{H}+p_{n} \mathbf{U}_{n} \mathbf{U}_{n}^{H}$, where $\mathbf{U}_{n}$ is orthonormal to $\mathbf{U}_{s}$.

Proof. The proof can be found in Appendix C.1.

It is obvious from (57) that, as the SNR approaches infinity, the CRB decays to zero for $D<|\mathbb{S}|$, which is consistent with the observation in [1].

For $D \geq|\mathbb{S}|$ and $\mathbf{V}_{\mathbb{S}}$ being full row rank, the asymptotic $\mathrm{CRB}$ expression can be given by

Theorem 4. If the $D$ uncorrelated sources have equal $S N R p / p_{n}, D \geq|\mathbb{S}|, \operatorname{rank}\left(\mathbf{V}_{\mathbb{S}}\right)=|\mathbb{S}|$, and $\operatorname{rank}\left(\mathbf{A}_{c}\right)=$ $2 D+1$, then for sufficiently large $S N R$, the CRB has an asymptotic expression which does not decay to zero as $S N R$ tends to infinity. Thus,

$$
\left.\operatorname{CRB}(\overline{\boldsymbol{\theta}})\right|_{\substack{\operatorname{rank}\left(\mathbf{V}_{\mathbb{S}}\right)=|\mathbb{S}| \\ \operatorname{large~SNR}}}=\frac{1}{4 \pi^{2} K} \mathbf{S}^{-1},
$$

where

$$
\begin{aligned}
\mathbf{S} & =\mathbf{G}_{\infty}^{H} \mathbf{\Pi}_{\mathbf{M}_{\infty} \mathbf{W}_{\mathbb{D}}}^{+\mathbf{G}_{\infty}} \\
\mathbf{M}_{\infty} & =\left(\mathbf{J}^{H}\left(\left(\mathbf{V}_{\mathbb{S}} \mathbf{V}_{\mathbb{S}}^{H}\right)^{-T} \otimes\left(\mathbf{V}_{\mathbb{S}} \mathbf{V}_{\mathbb{S}}^{H}\right)^{-1}\right) \mathbf{J}\right)^{\frac{1}{2}}, \\
\mathbf{G}_{\infty} & =\mathbf{M}_{\infty}(\operatorname{diag}(\mathbb{D})) \mathbf{V}_{\mathbb{D}}
\end{aligned}
$$

Here $\mathbf{W}_{\mathbb{D}}^{H} \mathbf{M}_{\infty}^{2} \mathbf{W}_{\mathbb{D}}$ and $\mathbf{S}$ are assumed to be nonsingular.

Proof. The proof can be found in Appendix C.2.

Theorem 4 also confirms what was empirically observed in [1], for $D \geq|\mathbb{S}|$. It will be demonstrated in Section 7.1 that the proposed CRB expression (49) indeed comes close to the asymptotic values (57) and (63). 


\section{Connection to the ULA Part of the Coarray}

It was observed from Property 3 that the difference coarray $\mathbb{D}$ has a direct impact on the singularity of the FIM. In this section, it will be shown that, if the difference coarray has certain structure, then the rank condition (48) is guaranteed for any choice of distinct DOAs. This can be regarded as a theoretical justification of the identifiability observations empirically made in Property 1 and 2 in Section 2.2.

Theorem 5. Let $\mathbb{U}$ be the central ULA segment of the difference coarray $\mathbb{D}$, as in Definition 2. Let $\mathbf{A}_{c}$ be the ACM matrix. If $D \leq(|\mathbb{U}|-1) / 2$, then $\operatorname{rank}\left(\mathbf{A}_{c}\right)=2 D+1$, for every $\overline{\boldsymbol{\theta}}=\left[\bar{\theta}_{1}, \bar{\theta}_{2}, \ldots, \bar{\theta}_{D}\right]^{T}$ such that $\bar{\theta}_{i} \neq \bar{\theta}_{j}$ for $i \neq j$.

Proof. The proof can be found in Appendix D.

Theorem 5 and Property 5 indicate that if $D \leq(|\mathbb{U}|-$ 1) $/ 2$, then the CRB expression approaches zero for large snapshots. This result is consistent with Property 1.

Corollary 1. If $D>(|\mathbb{D}|-1) / 2$, then for any choice of $D$ distinct DOAs, we have $\operatorname{rank}\left(\mathbf{A}_{c}\right)<2 D+1$, and the FIM is singular.

Proof. If $D>(|\mathbb{D}|-1) / 2$, then for any choice of $\overline{\boldsymbol{\theta}}$, the ACM matrix $\mathbf{A}_{c}$ becomes a fat matrix. This proves the Corollary.

Note that Corollary 1 explains the observation given in Property 2. Finally, when

$$
(|\mathbb{U}|-1) / 2<D \leq(|\mathbb{D}|-1) / 2,
$$

it is unclear whether the rank condition (48) holds true or not. For some choices of the DOA values, the rank condition (48) holds and for some values it does not. So in the regime (64), whether the FIM is nonsingular and whether the CRB exists depends on the specific values of the DOA.

\section{Numerical Examples}

\subsection{The proposed CRB expression versus snapshots and SNR}

Our first numerical example examines Property 5, Theorem 3, and Theorem 4. Consider a nested array with $N_{1}=N_{2}=2$, so that the sensor locations $\mathbb{S}=\{1,2,3,6\}$ and the difference coarray becomes $\mathbb{D}=\{-5, \ldots, 5\}$. As a result, the total number of sensors is 4 while the maximum number of identifiable sources is 5 . The equalpower sources are located at $\bar{\theta}_{i}=-0.49+0.9(i-1) / D$ for $i=1,2, \ldots, D$. It can be shown that these parameters indeed satisfy the rank condition (48), so that, the proposed CRB expression is valid.

Fig. 2 plots the proposed CRB expression for $\bar{\theta}_{1}$ as a function of snapshots, with $20 \mathrm{~dB}$ SNR. It can be observed that this expression is inversely proportional to the number

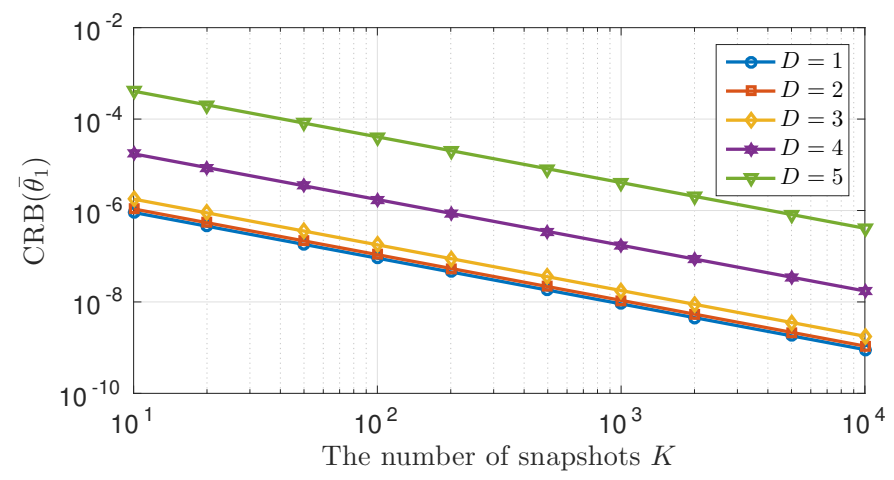

Figure 2: The dependence of the proposed CRB expression on snapshots for various numbers of sources $D$. The array configuration is the nested array with $N_{1}=N_{2}=2$ so that the sensor locations are $\mathbb{S}=\{1,2,3,6\}$. The equal-power sources are located at $\bar{\theta}_{i}=-0.49+0.9(i-1) / D$ for $i=1,2, \ldots, D$. SNR is $20 \mathrm{~dB}$.

of snapshots $K$, which verifies Property 5. These curves also depend on the number of sources $D$. In this specific example, these CRBs increase with $D$, which suggests that if there are more sources, it is more difficult to estimate $\bar{\theta}_{1}$ accurately.

Fig. 3(a) and (b) display the relationship between the proposed CRB expression and the SNR for 500 snapshots. Fig. 3(a) shows that if $D<|\mathbb{S}|=4$, the CRBs decrease with the SNR. For $D \geq|\mathbb{S}|=4$, the CRBs saturate when the SNR is over 20dB, as indicated in Fig. 3(b). These phenomena are consistent with what was observed experimentally in [1]. Furthermore, the dashed lines in Fig. 3(a) and (b) demonstrate that, for large SNR, the CRBs indeed converge to the asymptotic CRB expressions, as presented in Theorem 3 and 4 .

\subsection{The proposed $C R B$ expression for $U L A, M R A$, nested arrays, coprime arrays, and super nested arrays}

In the following simulations, consider the following five array configurations: uniform linear arrays (ULA) [102], minimum redundancy arrays (MRA) [61], nested arrays with $N_{1}=N_{2}=5$ [63], coprime arrays with $M=3, N=$ 5 [98], and second-order super nested arrays with $N_{1}=$ $N_{2}=5$ [51]. The sensor locations for these arrays are given by

$$
\begin{aligned}
\mathbb{S}_{\mathrm{ULA}} & =\{0,1,2,3,4,5,6,7,8,9\}, \\
\mathbb{S}_{\mathrm{MRA}} & =\{0,1,3,6,13,20,27,31,35,36\}, \\
\mathbb{S}_{\text {nested }} & =\{1,2,3,4,5,6,12,18,24,30\}, \\
\mathbb{S}_{\text {coprime }} & =\{0,3,5,6,9,10,12,15,20,25\}, \\
\mathbb{S}_{\text {super nested }} & =\{1,3,5,8,10,12,18,24,29,30\} .
\end{aligned}
$$

In each array, the total number of sensors is 10 . The difference coarray is listed as follows:

$$
\begin{aligned}
& \mathbb{D}_{\text {ULA }}=\{0, \pm 1, \ldots, \pm 9\} \\
& \mathbb{D}_{\text {MRA }}=\{0, \pm 1, \ldots, \pm 36\} \\
& \mathbb{D}_{\text {nested }}=\{0, \pm 1, \ldots, \pm 29\}
\end{aligned}
$$


(a)

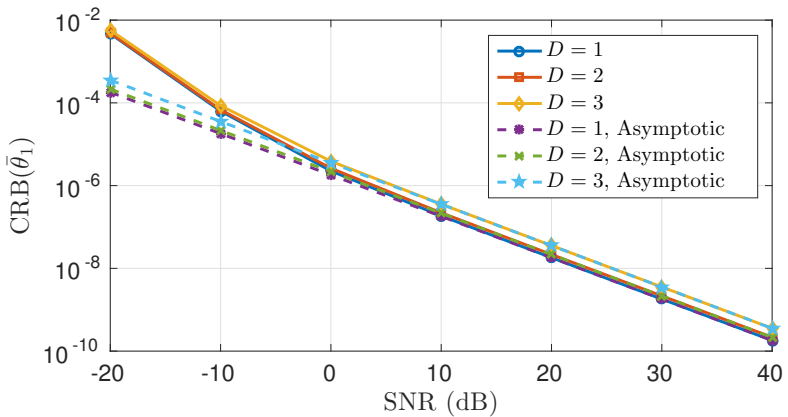

(b)

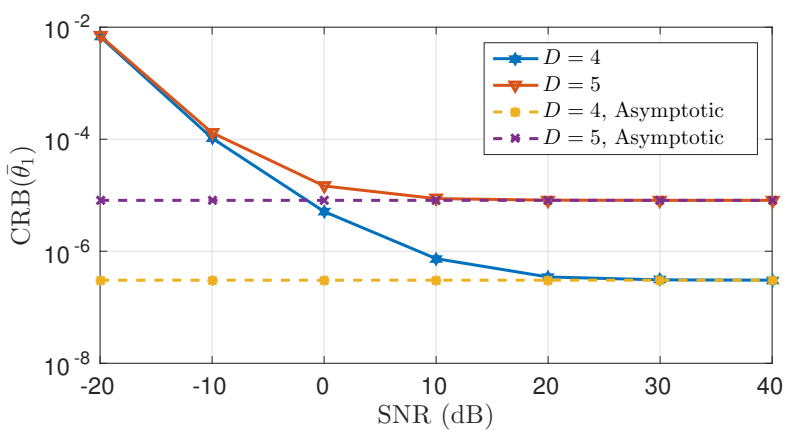

Figure 3: The dependence of the proposed CRB expression on SNR for (a) $D<|\mathbb{S}|=4$ and (b) $D \geq|\mathbb{S}|=4$. The array configuration is the nested array with $N_{1}=N_{2}=2$ so that the sensor locations are $\mathbb{S}=\{1,2,3,6\}$. The equal-power sources are located at $\bar{\theta}_{i}=$ $-0.49+0.9(i-1) / D$ for $i=1,2, \ldots, D$. The number of snapshots $K$ is 500 .

(a)

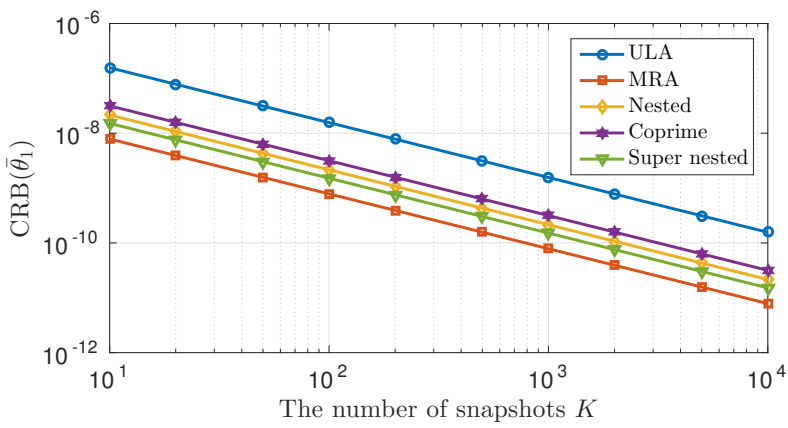

(b)

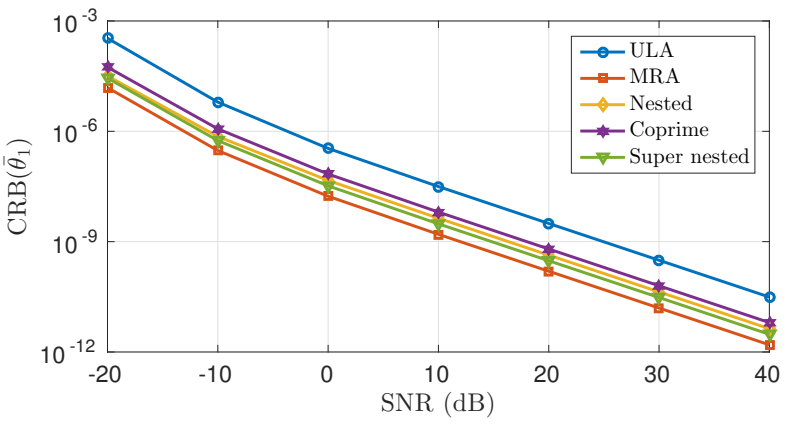

Figure 4: The dependence of the proposed CRB on (a) snapshots and (b) SNR for ULA, MRA, nested arrays, coprime arrays, and super nested arrays. The total number of sensors is 10 and the sensor locations are given in (65) to (68). The number of sources is $D=3$ (fewer sources than sensors) and the sources are located at $\bar{\theta}_{i}=-0.49+0.99(i-1) / D$ for $i=1,2, \ldots, D$. For (a), the SNR is $20 \mathrm{~dB}$ while for (b) the number of snapshots $K$ is 500 . (a)

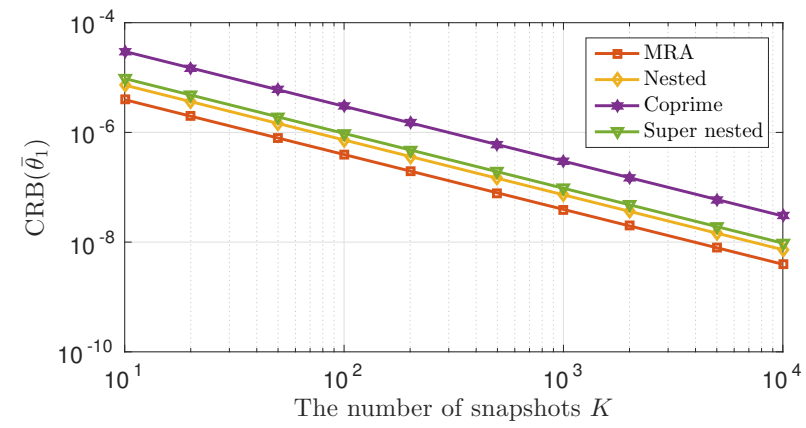

(b)

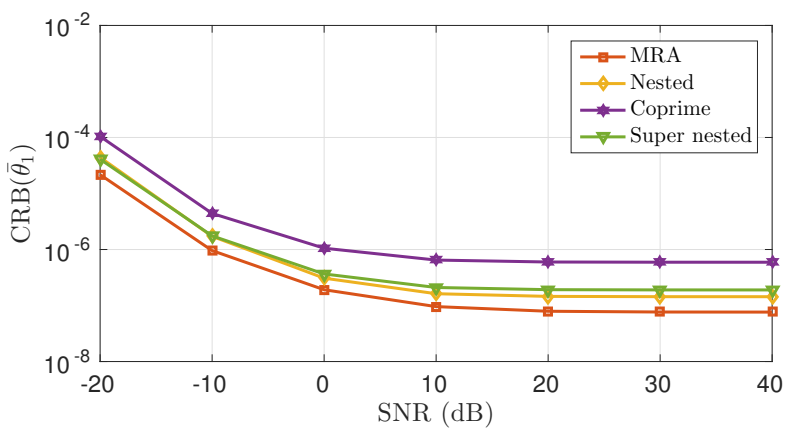

Figure 5: The dependence of the proposed CRB on (a) snapshots and (b) SNR for MRA, nested arrays, coprime arrays, and super nested arrays. The total number of sensors is 10 and the sensor locations are given in (65) to (68). The number of sources is $D=17$ (more sources than sensors) and the sources are located at $\bar{\theta}_{i}=$ $-0.49+0.99(i-1) / D$ for $i=1,2, \ldots, D$. For (a), the SNR is $20 \mathrm{~dB}$ while for (b) the number of snapshots $K$ is 500 .

Table 2: Identifiable/non-identifiable regions for coarray MUSIC.

\begin{tabular}{cccc}
\hline & Identifiable & $\begin{array}{c}\text { Cannot } \\
\text { judge }\end{array}$ & $\begin{array}{c}\text { Non- } \\
\text { identifiable }\end{array}$ \\
\hline ULA (65) & $1 \leq D \leq 9$ & - & $10 \leq D$ \\
MRA (66) & $1 \leq D \leq 36$ & - & $37 \leq D$ \\
Nested (67) & $1 \leq D \leq 29$ & - & $30 \leq D$ \\
Coprime (68) & $1 \leq D \leq 17$ & $18 \leq D \leq 21$ & $22 \leq D$ \\
Super nested & $1 \leq D \leq 29$ & - & $30 \leq D$ \\
$(69)$ & & &
\end{tabular}

$$
\begin{aligned}
\mathbb{D}_{\text {coprime }} & =\{0, \pm 1, \ldots, \pm 17, \pm 19, \pm 20, \pm 22, \pm 25\} \\
\mathbb{D}_{\text {super nested }} & =\{0, \pm 1, \ldots, \pm 29\}
\end{aligned}
$$

According to Property 1 and 2, the identifiability capabilities of coarray MUSIC are summarized in Table 2.

Fig. 4 compares the CRB for DOA estimation over ULA, MRA, nested arrays, coprime arrays, and super nested arrays if there are fewer sources $(D=3)$ than sensors $(|\mathbb{S}|=10)$. The equal-power sources are located at $\bar{\theta}_{i}=-0.49+0.99(i-1) / D$ for $i=1,2, \ldots, D$, where the number of sources $D$ is 3 . According to Table 2, all of these arrays can identify such sources using coarray MUSIC because $D<|\mathbb{S}|$. Fig. 4(a) depicts the CRBs in terms of the number of snapshots $K$ with $20 \mathrm{~dB}$ SNR while Fig. 4(b) shows the dependence of the CRBs on SNR for 500 


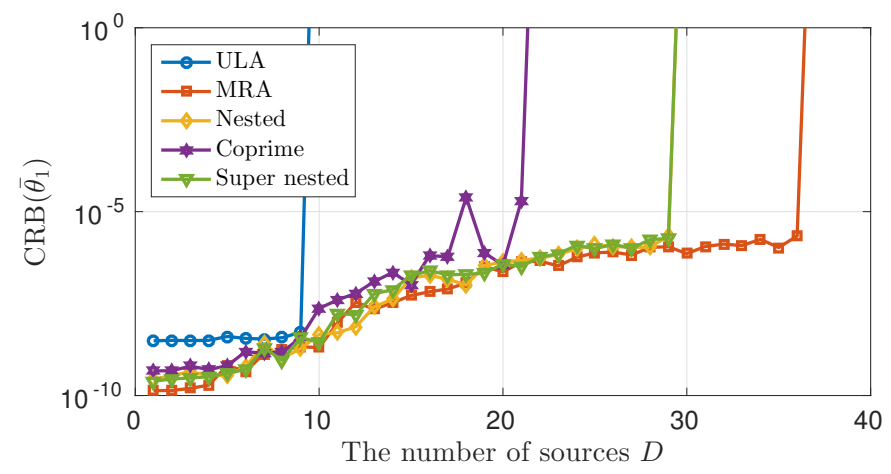

Figure 6: The dependence of the proposed CRB on the number of sources $D$ for various array configurations. The equal-power sources are located at $\bar{\theta}_{i}=-0.49+0.99(i-1) / D$ for $i=1,2, \ldots, D$. The number of snapshots $K$ is 500 and SNR is $20 \mathrm{~dB}$.

snapshots. It can be inferred that, for fixed $K$ and SNR, the least CRB is exhibited by MRA, followed by the super nested array, then the nested array, then the coprime array, and finally ULA. This ranking is consistent with the empirical observation that the estimation error decreases with the increasing size of the difference coarray $[1,61,63,64,99]$. In particular, the size of the difference coarray is 73 for MRA, 59 for the super nested array and the nested array, 43 for the coprime array, and 19 for ULA.

Fig. 5 illustrates the CRB for MRA, nested arrays, coprime arrays, and super nested arrays if there are more sources $(D=17)$ than sensors $(|\mathbb{S}|=10)$. The remaining parameters are identical to those in Fig. 4. The least CRB is now enjoyed by MRA, followed by the nested array, the super nested array, and finally the coprime array. Note that the CRB for ULA is divergent since the number of sources $D=17$ resides in the non-identifiable regime, as indicated in Table 2. Another obervation is that, in this example, the coprime array has the largest CRB. It is because the number of sources $D=17$ is the upper limit of the identifiable region for the coprime array, while the number of identifiable sources for the remaining three arrays is larger than 17 . Hence, the estimation performance for the coprime array is worst among the others.

\subsection{The proposed CRB expression versus the number of sources}

Next, the maximum number of detectable sources for ULA, MRA, nested arrays, coprime arrays, and super nested arrays is investigated. The sensor locations for these arrays are listed from (65) to (69). The normalized DOAs for $D$ equal-power sources are $\bar{\theta}_{i}=-0.49+$ $0.99(i-1) / D$ for $i=1,2, \ldots, D$. The SNR is $20 \mathrm{~dB}$ and the number of snapshots is 500. According to Property 1 and 2, the identifiability capabilities of coarray MUSIC are summarized in Table 2.

Fig. 6 evaluates the proposed CRB expression with respect to the number of sources $D$ for these array configurations. It can be observed that the identifiability, as shown in Table 2, is actually consistent with the proposed CRB (a)

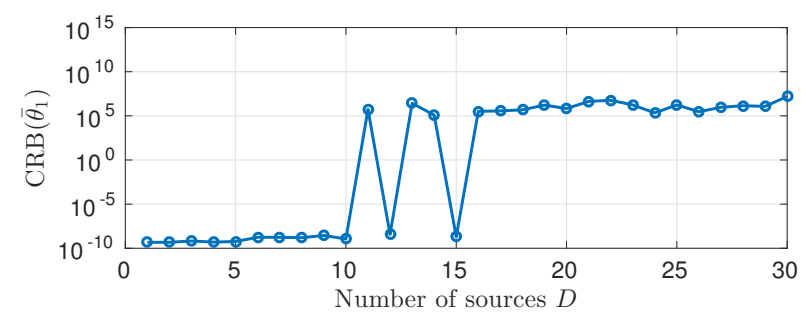

(b)
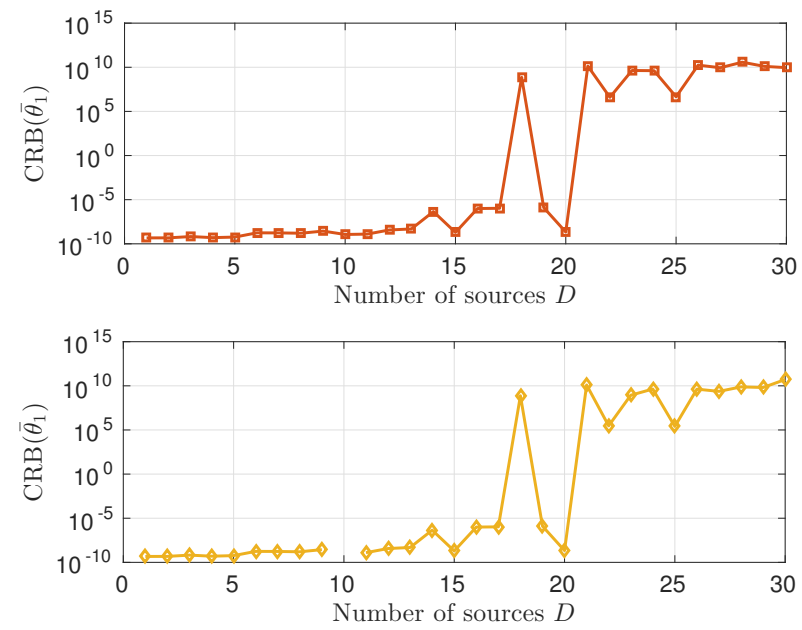

(d)

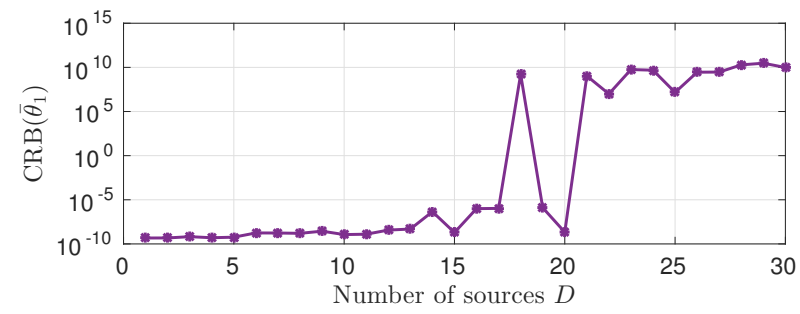

Figure 7: The CRB expressions versus the number of sources $D$ for a coprime array. (a) The stochastic CRB expression [91], (b) the CRB which is evaluated numerically by Abramovich et al. [1], (c) Jansson et al.'s CRB expression [40], and (d) the proposed CRB expression, as in Theorem 2. The coprime array with $M=3, N=5$ has sensor locations as in (68) and the difference coarray as in (73). The number of sensors $|\mathbb{S}|=10$. The equal-power sources are located at $\bar{\theta}_{i}=-0.48+(i-1) / D$ for $i=1,2, \ldots, D$. The number of snapshots $K$ is 500 and SNR is $20 \mathrm{~dB}$.

expression. Each CRB curve diverges for $D$ greater than a certain threshold, which can be inferred from the nonidentifiable regimes in Table 2. As an example, for the coprime array with $1 \leq D \leq 17$, the associated CRB expressions are small, which match the identifiability of coarray MUSIC in Property 1. On the other hand, for $D \geq 22$, the CRB expressions become divergent, which is consistent with non-identifiability of coarray MUSIC (Property 2 ). In the region $18 \leq D \leq 21$ which corresponds to the regime (64), the existence or otherwise of CRB is inconclusive. In this example, the CRB is small but in an example in Section 7.4, we will see that it is divergent.

Fig. 6 also elaborates the discussion, given earlier in Section 5, on the associated CRB expression for two sparse arrays $\mathbb{S}_{1}$ and $\mathbb{S}_{2}$ with the same difference coarray $\mathbb{D}$. Consider the CRBs for the nested array and the super nested array. It can be seen that both CRBs are convergent for 
$1 \leq D \leq 29$ and divergent for $D \geq 30$, even if the physical array configurations are different. This behavior is truly compatible with the discussion in Section 5.

\subsection{Comparison between the well-known CRB expres- sions and the proposed expression}

In this subsection, the coprime array with $M=3, N=5$ is considered, where the sensor locations are given in (68). The SNR is $20 \mathrm{~dB}$ and the number of snapshots is $K=500$. The sources have normalized DOAs $\bar{\theta}_{i}=-0.48+(i-1) / D$ for $i=1,2, \ldots, D$, which is different from those in Section 7.3 .

Fig. 7 depicts several different CRB expressions: (a) the stochastic CRB expression of [91], (b) the CRB which is evaluated numerically by Abramovich et al. [1], (c) Jansson et al.'s CRB expression [40], and (d) the proposed CRB expression, as in Theorem 2. First of all, the stochastic CRB expression of [91] is valid only when $D<|\mathbb{S}|$, as discussed in Section 4.1. Hence, it cannot be used to derive conclusions about identifiability in the regime $D \geq|\mathbb{S}|$. This is indeed seen in Fig. 7(a) where the CRB of [91] diverges for $D \geq|\mathbb{S}|=10$, even though this is still an identifiable regime according to Table 2 .

Abramovich et al.'s CRB expression, in Fig. 7(b), is calculated numerically from the FIM. Jansson et al.'s CRB expression, as shown in Fig. 7(c), is consistent with the identifiable and non-identifiable regions in Table 2, except for $D=|\mathbb{S}|=10$. It is because the appearance of $|\mathbb{S}|-D$ in the denominator of (32) makes the whole expression invalid. Furthermore, if $D>|\mathbb{S}|=10$, even though $\boldsymbol{\Pi}_{\mathbf{V}_{\mathbb{S}}}$ is undefined, we still calculate Jansson et al.'s CRB expression (31) numerically and it resembles the plot given in Fig. 7(b).

Finally, the newly proposed CRB expression (49), as plotted in Fig. 7(d), fully agrees with Jansson et al.'s CRB expression for $D<|\mathbb{S}|=10$ and Abramovich et al.'s for any $D$. Unlike Jansson et al.'s expression, the new expression can also be evaluated for $D=|\mathbb{S}|=10$. Furthermore, the proposed CRB expression is in agreement with the identifiablity results for every $D$ in Table 2 . This example also justifies the relations among Abramovich et al.'s, Jansson et al.'s, and the proposed CRB expression, as discussion in Section 4.3.

Note that in the example, the proposed CRB expression becomes divergent at $D=18$, as seen in Fig. $7(\mathrm{~d})$. It is because the rank of the ACM matrix is $35<2 D+1=37$, which violates the rank condition (48). Hence, these particular DOAs lead to a singular FIM, as stated in Theorem 1. This example also shows that, in the "unknown" region of Table 2, which is $18 \leq D \leq 21$, the FIM could be singular or nonsingular, depending on the DOAs.

\section{Concluding Remarks}

In this paper, we derived a new expression for the CRB of DOA estimates using linear arrays. The expression is especially useful in the case of sparse arrays such as nested arrays, coprime arrays, or MRAs, which can identify many more sources than the number of sensors. The conditions for validity of the expression are expressed in terms of the rank of an augmented coarray manifold matrix. The expression is valid for up to $O\left(N^{2}\right)$ sources where $N$ is the number of sensors. The precise details depend on the array configuration. We found that considerable insights regarding the behavior of sparse arrays can be gained from these expressions. All results were derived for uncorrelated sources, and only the estimation of source DOAs were considered, and not the source powers. In the future, it will be of interest to extend the results of this paper to the case where source powers are also parameters of interest. Extension to correlated sources will be of future interest as well.

\section{Appendix A. Derivation to the proposed CRB ex- pression}

Appendix A.1. Connection to the difference coarray

To simplify the CRB, it can be inferred from (38) that

$$
\begin{aligned}
\mathbf{r}_{\mathbb{S}} & =\operatorname{vec}\left(\sum_{i=1}^{D} p_{i} \mathbf{v}_{\mathbb{S}}\left(\bar{\theta}_{i}\right) \mathbf{v}_{\mathbb{S}}^{H}\left(\bar{\theta}_{i}\right)+p_{n} \mathbf{I}\right) \\
& =\sum_{i=1}^{D} p_{i} \operatorname{vec}\left(\mathbf{v}_{\mathbb{S}}\left(\bar{\theta}_{i}\right) \mathbf{v}_{\mathbb{S}}^{H}\left(\bar{\theta}_{i}\right)\right)+p_{n} \operatorname{vec}(\mathbf{I}) \\
& =\sum_{i=1}^{D} p_{i} \mathbf{v}_{\mathbb{S}}^{*}\left(\bar{\theta}_{i}\right) \otimes \mathbf{v}_{\mathbb{S}}\left(\bar{\theta}_{i}\right)+p_{n} \operatorname{vec}(\mathbf{I}),
\end{aligned}
$$

since $\operatorname{vec}\left(\mathbf{a} \mathbf{b}^{T}\right)=\mathbf{b} \otimes \mathbf{a}[56]$. It is useful to express $\mathbf{v}_{\mathbb{S}}^{*}\left(\bar{\theta}_{i}\right) \otimes$ $\mathbf{v}_{\mathbb{S}}\left(\bar{\theta}_{i}\right)$ in terms of the difference coarray manifold vector $\mathbf{v}_{\mathbb{D}}\left(\bar{\theta}_{i}\right)$ using a matrix $\mathbf{J}$ as follows:

$$
\mathbf{v}_{\mathbb{S}}^{*}\left(\bar{\theta}_{i}\right) \otimes \mathbf{v}_{\mathbb{S}}\left(\bar{\theta}_{i}\right)=\mathbf{J} \mathbf{v}_{\mathbb{D}}\left(\bar{\theta}_{i}\right)
$$

The appropriate matrix $\mathbf{J}$ for this is given in Appendix B. It is shown in Appendix B that $\mathbf{J}$ has full column rank, which leads to the following corollary:

Corollary 2. $\mathbf{J}^{H}\left(\mathbf{R}_{\mathbb{S}}^{T} \otimes \mathbf{R}_{\mathbb{S}}\right)^{-1} \mathbf{J}$ is positive definite. Therefore, it has a positive definite square root $\mathbf{M}=$ $\left(\mathbf{J}^{H}\left(\mathbf{R}_{\mathbb{S}}^{T} \otimes \mathbf{R}_{\mathbb{S}}\right)^{-1} \mathbf{J}\right)^{\frac{1}{2}}$.

Proof. Since $p_{1}, \ldots, p_{D}, p_{n}>0, \mathbf{R}_{\mathbb{S}}$ and $\mathbf{R}_{\mathbb{S}}^{T}$ are both positive definite, implying $\left(\mathbf{R}_{\mathbb{S}}^{T} \otimes \mathbf{R}_{\mathbb{S}}\right)^{-1}$ is also positive definite [56]. Hence, $\mathbf{J}^{H}\left(\mathbf{R}_{\mathbb{S}}^{T} \otimes \mathbf{R}_{\mathbb{S}}\right)^{-1} \mathbf{J}$ is positive definite $[37,56]$.

Property 7 and Corollary 3, both given later in Ap- 
pendix B, simplify (A.1) as

$$
\begin{aligned}
& \mathbf{r}_{\mathbb{S}}=\mathbf{J}_{\mathbf{x}_{\mathbb{D}}}=\mathbf{J}\left(\mathbf{V}_{\mathbb{D}} \mathbf{p}+p_{n} \mathbf{e}_{0}\right)=\mathbf{J} \mathbf{W}_{\mathbb{D}}\left[\begin{array}{c}
\mathbf{p} \\
p_{n}
\end{array}\right],
\end{aligned}
$$

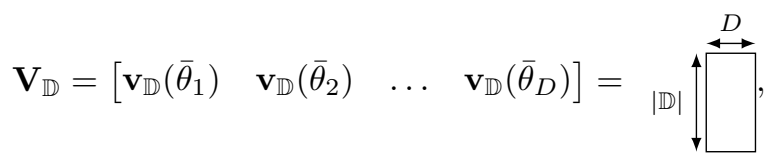

$$
\begin{aligned}
& \mathbf{W}_{\mathbb{D}}=\left[\begin{array}{ll}
\mathbf{V}_{\mathbb{D}} & \mathbf{e}_{0}
\end{array}\right]=\stackrel{\mathbb{D} \mid}{\stackrel{\Delta+1}{\rightleftarrows},},
\end{aligned}
$$

and $\mathbf{p}=\left[p_{1}, p_{2}, \ldots, p_{D}\right]^{T}$.

\section{Appendix A.2. Proof of Lemma 1}

(Sufficiency) If $\mathbf{D}$ is nonsingular, $\mathbf{F}$ can always be decomposed as [37, 56, 101] [97, Problem A.18]

$$
\begin{aligned}
\mathbf{F} & =\left[\begin{array}{cc}
\mathbf{A} & \mathbf{B} \\
\mathbf{B}^{H} & \mathbf{D}
\end{array}\right] \\
& =\left[\begin{array}{cc}
\mathbf{I} & \mathbf{B D}^{-1} \\
\mathbf{0} & \mathbf{I}
\end{array}\right]\left[\begin{array}{cc}
\mathbf{A}-\mathbf{B D}^{-1} \mathbf{B}^{H} & \mathbf{0} \\
\mathbf{0} & \mathbf{D}
\end{array}\right]\left[\begin{array}{cc}
\mathbf{I} & \mathbf{0} \\
\mathbf{D}^{-1} \mathbf{B}^{H} & \mathbf{I}
\end{array}\right]
\end{aligned}
$$

Taking the determinant on both sides of (A.7) leads to

$$
\operatorname{det}(\mathbf{F})=\operatorname{det}\left(\mathbf{A}-\mathbf{B D}^{-1} \mathbf{B}^{H}\right) \operatorname{det}(\mathbf{D}) .
$$

If $\mathbf{A}-\mathbf{B D}^{-1} \mathbf{B}^{H}$ is also nonsingular, then $\operatorname{det}(\mathbf{A}-$ $\left.\mathbf{B D}^{-1} \mathbf{B}^{H}\right) \neq 0$ and $\operatorname{det}(\mathbf{F}) \neq 0$. Hence, $\mathbf{F}$ is nonsingular.

(Necessity) Suppose $\mathbf{D}$ is nonsingular and $\mathbf{A}-\mathbf{B D}^{-1} \mathbf{B}^{H}$ is singular. Then $\operatorname{det}\left(\mathbf{A}-\mathbf{B D}^{-1} \mathbf{B}^{H}\right)=0$. Eq. (A.8) becomes $\operatorname{det}(\mathbf{F})=0$ hence $\mathbf{F}$ is singular.

If $\mathbf{D}$ is singular, then $\operatorname{det}(\mathbf{D})=0$. It is well-known that a Hermitian matrix is positive definite if and only if all the leading principal minors are positive [97, Fact A.6.3]. Since $\operatorname{det}(\mathbf{D})$ is a leading principal minor, it follows trivially that $\mathbf{F}$ is not positive definite. This concludes the proof.

\section{Appendix A.3. Proof of Lemma 2}

(Sufficiency) According to (42) and (A.3), $\boldsymbol{\Delta}^{H} \boldsymbol{\Delta}=$ $\mathbf{W}_{\mathbb{D}}^{H} \mathbf{J}^{H}\left(\mathbf{R}_{\mathbb{S}}^{T} \otimes \mathbf{R}_{\mathbb{S}}\right)^{-1} \mathbf{J W}_{\mathbb{D}}$. Since $\operatorname{rank}\left(\mathbf{W}_{\mathbb{D}}\right)=D+1$, it follows from Corollary 2 that $\boldsymbol{\Delta}^{H} \boldsymbol{\Delta}$ is positive definite.

(Necessity) If $\operatorname{rank}\left(\mathbf{W}_{\mathbb{D}}\right)<D+1$, then there exists a non-zero vector $\mathbf{u}$ such that $\mathbf{W}_{\mathbb{D}} \mathbf{u}=\mathbf{0}$. It can be deduced that

$$
\mathbf{u}^{H}\left(\boldsymbol{\Delta}^{H} \boldsymbol{\Delta}\right) \mathbf{u}=\left(\mathbf{W}_{\mathbb{D}} \mathbf{u}\right)^{H} \mathbf{J}^{H}\left(\mathbf{R}_{\mathbb{S}}^{T} \otimes \mathbf{R}_{\mathbb{S}}\right)^{-1} \mathbf{J}\left(\mathbf{W}_{\mathbb{D}} \mathbf{u}\right)=0,
$$

implying that $\boldsymbol{\Delta}^{H} \boldsymbol{\Delta}$ is not positive definite.
Appendix A.4. Proof of Lemma 3

(Sufficiency) Combining (A.3) and (41) yields

$$
\begin{aligned}
& \mathbf{G}=\left(\mathbf{R}_{\mathbb{S}}^{T} \otimes \mathbf{R}_{\mathbb{S}}\right)^{-\frac{1}{2}} \mathbf{J}\left[p_{1} \frac{\partial \mathbf{v}_{\mathbb{N}}\left(\bar{\theta}_{1}\right)}{\partial \bar{\theta}_{1}} \quad \ldots \quad p_{D} \frac{\partial \mathbf{v}_{\mathbb{D}}\left(\bar{\theta}_{D}\right)}{\partial \bar{\theta}_{D}}\right] \\
& =j 2 \pi\left(\mathbf{R}_{\mathbb{S}}^{T} \otimes \mathbf{R}_{\mathbb{S}}\right)^{-\frac{1}{2}} \mathbf{J} \\
& \times \operatorname{diag}(\mathbb{D})\left[p_{1} \mathbf{v}_{\mathbb{D}}\left(\bar{\theta}_{1}\right) \ldots p_{D} \mathbf{v}_{\mathbb{D}}\left(\bar{\theta}_{D}\right)\right] \\
& =j 2 \pi\left(\mathbf{R}_{\mathbb{S}}^{T} \otimes \mathbf{R}_{\mathbb{S}}\right)^{-\frac{1}{2}} \mathbf{J}(\operatorname{diag}(\mathbb{D})) \mathbf{V}_{\mathbb{D}} \mathbf{P},
\end{aligned}
$$

where $\mathbf{P}=\operatorname{diag}\left(p_{1}, p_{2}, \ldots, p_{D}\right)$. Similarly, (42) and (A.3) lead to

$$
\begin{aligned}
& \boldsymbol{\Delta}=\left(\mathbf{R}_{\mathbb{S}}^{T} \otimes \mathbf{R}_{\mathbb{S}}\right)^{-\frac{1}{2}} \mathbf{J}\left[\mathbf{v}_{\mathbb{D}}\left(\bar{\theta}_{1}\right) \quad \ldots \quad \mathbf{v}_{\mathbb{D}}\left(\bar{\theta}_{D}\right) \quad \mathbf{e}_{0}\right] \\
& =\left(\mathbf{R}_{\mathbb{S}}^{T} \otimes \mathbf{R}_{\mathbb{S}}\right)^{-\frac{1}{2}} \mathbf{J} \mathbf{W}_{\mathbb{D}} .
\end{aligned}
$$

Substituting (A.9) and (A.10) into $\mathbf{G}^{H} \mathbf{\Pi}_{\Delta}^{\perp} \mathbf{G} /\left(4 \pi^{2}\right)$ gives (A.11), where the matrix $\mathbf{M}$ is defined in Corollary 2.

Let $\mathbf{u} \in \mathbb{C}^{D}$. Since the projection matrix $\Pi_{\mathbf{M} \mathbf{W}_{\mathrm{D}}}^{\perp}$ is Hermitian and idempotent [37, 56], it can be deduced from (A.11) that

$$
\mathbf{u}^{H}\left(\frac{\mathbf{G}^{H} \boldsymbol{\Pi}_{\Delta}^{\perp} \mathbf{G}}{4 \pi^{2}}\right) \mathbf{u}=\left\|\boldsymbol{\Pi}_{\mathbf{M} \mathbf{W}_{\mathbb{D}}}^{\perp} \mathbf{G}_{0} \mathbf{u}\right\|_{2}^{2} \geq 0 .
$$

The equality holds only if $\mathbf{\Pi}_{\mathbf{M} \mathbf{W}_{\mathbb{D}}}^{\perp} \mathbf{G}_{0} \mathbf{u}=\mathbf{0}$, i.e., only if there exits a vector $\mathbf{v} \in \mathbb{C}^{D+1}$ such that

$$
\mathbf{M}(\operatorname{diag}(\mathbb{D})) \mathbf{V}_{\mathbb{D}} \mathbf{P u}=\mathbf{M} \mathbf{W}_{\mathbb{D}} \mathbf{v}
$$

Since $\mathbf{M}$ is positive definite, (A.12) can be expressed as

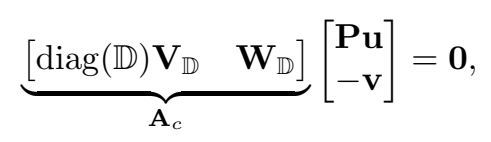

where $\mathbf{A}_{c}$ is the ACM matrix, as defined in Definition 4 . If $\operatorname{rank}\left(\mathbf{A}_{c}\right)=2 D+1$, then $\mathbf{P u}=\mathbf{0}$, implying $\mathbf{u}=\mathbf{0}$. As a result, $\mathbf{G}^{H} \boldsymbol{\Pi}_{\Delta}^{\perp} \mathbf{G}$ is positive definite.

(Necessity) If $\operatorname{rank}\left(\mathbf{A}_{c}\right)<2 D+1$, then there exists $\mathbf{a} \in$ $\mathbb{C}^{D}$ and $\mathbf{b} \in \mathbb{C}^{D+1}$ such that $\left[\mathbf{a}^{T}, \mathbf{b}^{T}\right]^{T} \neq \mathbf{0}$ and

$$
\operatorname{diag}(\mathbb{D}) \mathbf{V}_{\mathbb{D}} \mathbf{a}+\mathbf{W}_{\mathbb{D}} \mathbf{b}=\mathbf{0} .
$$

Left multiplying (A.13) by $\mathbf{M}$ leads to an expression similar to (A.12):

$$
\left(\mathbf{M}(\operatorname{diag}(\mathbb{D})) \mathbf{V}_{\mathbb{D}} \mathbf{P}\right)\left(\mathbf{P}^{-1} \mathbf{a}\right)=\left(\mathbf{M W}_{\mathbb{D}}\right)(-\mathbf{b}),
$$

where $\mathbf{P}=\operatorname{diag}\left(p_{1}, \ldots, p_{D}\right)$ is positive definite. If $\mathbf{a} \neq \mathbf{0}$, then $\mathbf{P}^{-1} \mathbf{a} \neq \mathbf{0}, \boldsymbol{\Pi}_{\mathbf{M} \mathbf{W}_{\mathbb{D}}}^{\perp} \mathbf{G}_{0}\left(\mathbf{P}^{-1} \mathbf{a}\right)=\mathbf{0}$, and $\mathbf{G}^{H} \boldsymbol{\Pi}_{\mathbf{\Delta}}^{\perp} \mathbf{G}$ is not positive definite. On the other hand, if $\mathbf{a}=\mathbf{0}$ and $\mathbf{b} \neq$ $\mathbf{0}$, then (A.14) becomes $\mathbf{W}_{\mathbb{D}} \mathbf{b}=\mathbf{0}$, which contradicts with the assumption that $\operatorname{rank}\left(\mathbf{W}_{\mathbb{D}}\right)=D+1$. These arguments complete the proof. 


$$
\begin{aligned}
\frac{\mathbf{G}^{H} \boldsymbol{\Pi}_{\boldsymbol{\Delta}}^{\perp} \mathbf{G}}{4 \pi^{2}} & =\mathbf{P}^{H} \mathbf{V}_{\mathbb{D}}^{H}(\operatorname{diag}(\mathbb{D}))^{H}\left[\mathbf{J}^{H}\left(\mathbf{R}_{\mathbb{S}}^{T} \otimes \mathbf{R}_{\mathbb{S}}\right)^{-\frac{1}{2}} \boldsymbol{\Pi}_{\left(\mathbf{R}_{\mathbb{S}}^{T} \otimes \mathbf{R}_{\mathbb{S}}\right)^{-\frac{1}{2}} \mathbf{J W}_{\mathbb{D}}}\left(\mathbf{R}_{\mathbb{S}}^{T} \otimes \mathbf{R}_{\mathbb{S}}\right)^{-\frac{1}{2}} \mathbf{J}\right](\operatorname{diag}(\mathbb{D})) \mathbf{V}_{\mathbb{D}} \mathbf{P} \\
& =\mathbf{P}^{H} \mathbf{V}_{\mathbb{D}}^{H}(\operatorname{diag}(\mathbb{D}))^{H}\left[\mathbf{M}^{H} \mathbf{M}-\mathbf{M}^{H}\left(\mathbf{M} \mathbf{W}_{\mathbb{D}}\right)\left[\left(\mathbf{M} \mathbf{W}_{\mathbb{D}}\right)^{H}\left(\mathbf{M} \mathbf{W}_{\mathbb{D}}\right)\right]^{-1}\left(\mathbf{M} \mathbf{W}_{\mathbb{D}}\right)^{H} \mathbf{M}\right](\operatorname{diag}(\mathbb{D})) \mathbf{V}_{\mathbb{D}} \mathbf{P} \\
& =\mathbf{G}_{0}^{H} \mathbf{\Pi}_{\mathbf{M} \mathbf{W}_{\mathbb{D}}}^{\perp} \mathbf{G}_{0}, \quad \text { where } \mathbf{G}_{0}=\mathbf{M}(\operatorname{diag}(\mathbb{D})) \mathbf{V}_{\mathbb{D}} \mathbf{P} .
\end{aligned}
$$

\section{Appendix B. Definition of $\mathbf{J}$}

Definition 5. The binary matrix $\mathbf{J}$ has size $|\mathbb{S}|^{2}$-by-| $|\mathbb{D}|$ such that the column of $\mathbf{J}$ associated with the difference $m$ is given by

$$
\langle\mathbf{J}\rangle_{:, m}=\operatorname{vec}(\mathbf{I}(m)), \quad m \in \mathbb{D},
$$

where the $|\mathbb{S}|$-by- $|\mathbb{S}|$ matrix $\mathbf{I}(m)$ satisfies

$$
\langle\mathbf{I}(m)\rangle_{n_{1}, n_{2}}=\left\{\begin{array}{ll}
1 & \text { if } n_{1}-n_{2}=m, \\
0 & \text { otherwise. }
\end{array} \quad n_{1}, n_{2} \in \mathbb{S} .\right.
$$

As an example of $\mathbf{J}$, if $\mathbb{S}=\{0,1,4\}$, then $\mathbb{D}=$ $\{-4,-3,-1,0,1,3,4\}$, and $\mathbf{I}(m)$ are

$$
\begin{aligned}
& \mathbf{I}(m=0)=\begin{array}{r}
n_{2}=0 \\
n_{1}=0 \\
1 \\
4
\end{array}\left[\begin{array}{lll}
1 & 0 & 0 \\
0 & 1 & 0 \\
0 & 0 & 1
\end{array}\right], \\
& \mathbf{I}(m=1)=\begin{array}{r}
n_{2}=0 \\
n_{1}=0 \\
1 \\
4
\end{array}\left[\begin{array}{lll}
0 & 0 & 4 \\
1 & 0 & 0 \\
0 & 0 & 0
\end{array}\right], \\
& \mathbf{I}(m=3)=\begin{array}{r}
n_{2}=0 \\
n_{1}=0 \\
1 \\
4
\end{array}\left[\begin{array}{lll}
0 & 1 & 4 \\
0 & 0 & 0 \\
0 & 1 & 0
\end{array}\right], \\
& \mathbf{I}(m=4)=\begin{array}{r}
n_{2}=0 \\
n_{1}=0 \\
1 \\
4
\end{array}\left[\begin{array}{lll}
0 & 0 & 0 \\
0 & 0 & 0 \\
1 & 0 & 0
\end{array}\right],
\end{aligned}
$$

and $\mathbf{I}(-1)=\mathbf{I}(1)^{T}, \mathbf{I}(-3)=\mathbf{I}(3)^{T}$, and $\mathbf{I}(-4)=\mathbf{I}(4)^{T}$. As a result, $\mathbf{J}$ is given by

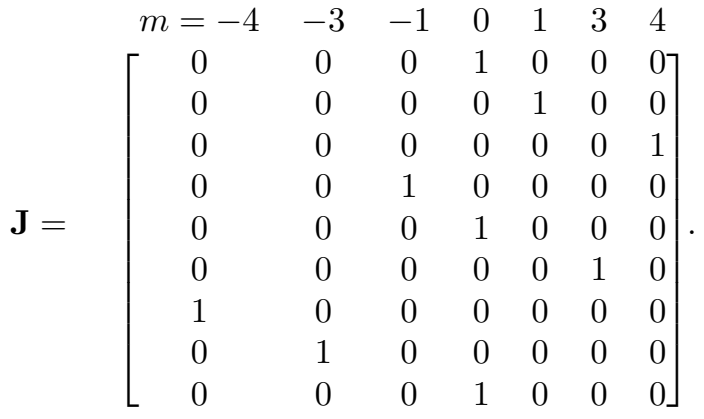

The following properties of $\mathbf{J}$ are useful:

Property 6. J has full column rank, i.e., $\operatorname{rank}(\mathbf{J})=|\mathbb{D}|$.

Proof. It suffices to prove that $\{\mathbf{I}(m)\}_{m \in \mathbb{D}}$ is a linearly independent set. Consider the linear equation $\sum_{m \in \mathbb{D}} c_{m} \mathbf{I}(m)=\mathbf{0}$. Suppose there exists $P \in \mathbb{D}$ such that $c_{P} \neq 0$. For any $n_{1}, n_{2} \in \mathbb{S}$ such that $n_{1}-n_{2}=P$, it can be deduced that

$$
\begin{aligned}
0 & =\langle\mathbf{0}\rangle_{n_{1}, n_{2}}=\left\langle\sum_{m \in \mathbb{D}} c_{m} \mathbf{I}(m)\right\rangle_{n_{1}, n_{2}} \\
& =\sum_{m \in \mathbb{D}} c_{m}\langle\mathbf{I}(m)\rangle_{n_{1}, n_{2}}=c_{P},
\end{aligned}
$$

which contradicts $c_{P} \neq 0$. Hence, the coefficients $c_{m}$ are all zero, implying $\mathbf{J}$ has full column rank.

Property 7. With $\mathbf{J}$ as in Definition 5 , we have $\mathbf{v}_{\mathbb{S}}^{*}\left(\bar{\theta}_{i}\right) \otimes$ $\mathbf{v}_{\mathbb{S}}\left(\bar{\theta}_{i}\right)=\mathbf{J}_{\mathbf{v}_{\mathbb{D}}}\left(\bar{\theta}_{i}\right)$.

Proof. Letting $\alpha_{i}=e^{j 2 \pi \bar{\theta}_{i}}$ and starting with the righthand side of the equation yield

$$
\mathbf{J}_{\mathbf{v}_{\mathbb{D}}}\left(\bar{\theta}_{i}\right)=\sum_{m \in \mathbb{D}} \operatorname{vec}(\mathbf{I}(m)) \alpha_{i}^{m}=\operatorname{vec}\left(\sum_{m \in \mathbb{D}} \mathbf{I}(m) \alpha_{i}^{m}\right) .
$$

The $\left(n_{1}, n_{2}\right)$ entry of $\sum_{m \in \mathbb{D}} \mathbf{I}(m) \alpha_{i}^{m}$ is

$$
\left\langle\sum_{m \in \mathbb{D}} \mathbf{I}(m) \alpha_{i}^{m}\right\rangle_{n_{1}, n_{2}}=\sum_{m \in \mathbb{D}}\langle\mathbf{I}(m)\rangle_{n_{1}, n_{2}} \alpha_{i}^{m}=\alpha_{i}^{n_{1}}\left(\alpha_{i}^{n_{2}}\right)^{*},
$$

so that $\sum_{m \in \mathbb{D}} \mathbf{I}(m) \alpha_{i}^{m}=\mathbf{v}_{\mathbb{S}}\left(\bar{\theta}_{i}\right) \mathbf{v}_{\mathbb{S}}^{H}\left(\bar{\theta}_{i}\right)$. Therefore, $\mathbf{J}_{\mathbf{v}_{\mathbb{D}}}\left(\bar{\theta}_{i}\right)=\operatorname{vec}\left(\mathbf{v}_{\mathbb{S}}\left(\bar{\theta}_{i}\right) \mathbf{v}_{\mathbb{S}}^{H}\left(\bar{\theta}_{i}\right)\right)=\mathbf{v}_{\mathbb{S}}^{*}\left(\bar{\theta}_{i}\right) \otimes \mathbf{v}_{\mathbb{S}}\left(\bar{\theta}_{i}\right)$, which proves the property.

Corollary 3. $\operatorname{vec}(\mathbf{I})=\mathbf{J e}_{0}$, where $\mathbf{e}_{0} \in\{0,1\}^{|\mathbb{D}|}$ satisfying

$$
\left\langle\mathbf{e}_{0}\right\rangle_{m}=\left\{\begin{array}{ll}
1, & \text { if } m=0, \\
0, & \text { otherwise, }
\end{array} \quad m \in \mathbb{D} .\right.
$$

\section{Appendix C. Proof of the asymptotic CRB ex- pression for large SNR}

Appendix C.1. Proof of Theorem 3

According to Property 4, the CRB expression depends on the SNR $p / p_{n}$, so without loss of generality, we set $p_{n}=$ 1. For sufficiently large SNR, $p$ is much greater than $p_{n}$, so that $\mathbf{R}_{\mathbb{S}}$ can be approximated by $p \mathbf{U}_{s} \boldsymbol{\Lambda} \mathbf{U}_{s}^{H}+\mathbf{U}_{n} \mathbf{U}_{n}^{H}$ and 
$\mathbf{R}_{\mathbb{S}}^{-1}$ approaches $p^{-1} \mathbf{U}_{s} \boldsymbol{\Lambda}^{-1} \mathbf{U}_{s}^{H}+\mathbf{U}_{n} \mathbf{U}_{n}^{H}$ asymptotically. The quantity $\mathbf{R}_{\mathbb{S}}^{-T} \otimes \mathbf{R}_{\mathbb{S}}^{-1}$ can be expressed as

$$
\mathbf{R}_{\mathbb{S}}^{-T} \otimes \mathbf{R}_{\mathbb{S}}^{-1}=\mathbf{A}+p^{-1} \mathbf{B}+p^{-2} \mathbf{C},
$$

where $\mathbf{A}, \mathbf{B}$, and $\mathbf{C}$ are defined as

$$
\begin{aligned}
\mathbf{A}= & \left(\mathbf{U}_{n} \mathbf{U}_{n}^{H}\right)^{T} \otimes\left(\mathbf{U}_{n} \mathbf{U}_{n}^{H}\right) \\
\mathbf{B}= & {\left[\left(\mathbf{U}_{s} \boldsymbol{\Lambda}^{-1} \mathbf{U}_{s}^{H}\right)^{T} \otimes\left(\mathbf{U}_{n} \mathbf{U}_{n}^{H}\right)\right.} \\
& \left.\quad+\left(\mathbf{U}_{n} \mathbf{U}_{n}^{H}\right)^{T} \otimes\left(\mathbf{U}_{s} \boldsymbol{\Lambda}^{-1} \mathbf{U}_{s}^{H}\right)\right], \\
\mathbf{C}= & \left(\mathbf{U}_{s} \boldsymbol{\Lambda}^{-1} \mathbf{U}_{s}^{H}\right)^{T} \otimes\left(\mathbf{U}_{s} \boldsymbol{\Lambda}^{-1} \mathbf{U}_{s}^{H}\right) .
\end{aligned}
$$

For large $p, \mathbf{R}_{\mathbb{S}}^{-T} \otimes \mathbf{R}_{\mathbb{S}}^{-1}$ can be approximated by

$$
\mathbf{R}_{\mathbb{S}}^{-T} \otimes \mathbf{R}_{\mathbb{S}}^{-1} \stackrel{\text { large } p}{\longrightarrow} \mathbf{A}+p^{-1} \mathbf{B} .
$$

The following corollaries show two identities regarding $\mathbf{A}$ :

Corollary 4. $\mathbf{A J}(\operatorname{diag}(\mathbb{D})) \mathbf{V}_{\mathbb{D}}=\mathbf{0}$, where $\mathbf{A}$ and $\mathbf{J}$ are given in (C.1) and Definition 5, respectively.

Proof. According to Definition 5, the $i$ th column of $\mathbf{A J}(\operatorname{diag}(\mathbb{D})) \mathbf{V}_{\mathbb{D}}$ can be expressed as

$$
\mathbf{A J}(\operatorname{diag}(\mathbb{D})) \mathbf{v}_{\mathbb{D}}\left(\bar{\theta}_{i}\right)=\mathbf{A v e c}\left(\sum_{m \in \mathbb{D}} \mathbf{I}(m) m \alpha_{i}^{m}\right),
$$

where $\alpha_{i}=e^{j 2 \pi \bar{\theta}_{i}}$. The matrix $\mathbf{I}(m)$ is defined in Definition 5 . The $\left(n_{1}, n_{2}\right)$ entry of $\sum_{m \in \mathbb{D}} \mathbf{I}(m) m \alpha_{i}^{m}$ becomes

$$
\left\langle\sum_{m \in \mathbb{D}} \mathbf{I}(m) m \alpha_{i}^{m}\right\rangle_{n_{1}, n_{2}}=n_{1} \alpha_{i}^{n_{1}}\left(\alpha_{i}^{n_{2}}\right)^{*}-\alpha_{i}^{n_{1}}\left(\alpha_{i}^{n_{2}}\right)^{*} n_{2},
$$

so that

$$
\begin{aligned}
& \sum_{m \in \mathbb{D}} \mathbf{I}(m) m \alpha_{i}^{m} \\
= & \operatorname{diag}(\mathbb{S}) \mathbf{v}_{\mathbb{S}}\left(\bar{\theta}_{i}\right) \mathbf{v}_{\mathbb{S}}^{H}\left(\bar{\theta}_{i}\right)-\mathbf{v}_{\mathbb{S}}\left(\bar{\theta}_{i}\right) \mathbf{v}_{\mathbb{S}}^{H}\left(\bar{\theta}_{i}\right)(\operatorname{diag}(\mathbb{S})) .
\end{aligned}
$$

Substituting (C.6) into (C.5) yields

$$
\begin{aligned}
\mathbf{A J}(\operatorname{diag}(\mathbb{D})) \mathbf{v}_{\mathbb{D}}\left(\bar{\theta}_{i}\right) \\
=\left[\left(\mathbf{U}_{n} \mathbf{U}_{n}^{H}\right)^{T} \otimes\left(\mathbf{U}_{n} \mathbf{U}_{n}^{H}\right)\right] \\
\quad \times \operatorname{vec}\left(\operatorname{diag}(\mathbb{S}) \mathbf{v}_{\mathbb{S}}\left(\bar{\theta}_{i}\right) \mathbf{v}_{\mathbb{S}}^{H}\left(\bar{\theta}_{i}\right)-\mathbf{v}_{\mathbb{S}}\left(\bar{\theta}_{i}\right) \mathbf{v}_{\mathbb{S}}^{H}\left(\bar{\theta}_{i}\right)(\operatorname{diag}(\mathbb{S}))\right) \\
=\operatorname{vec}(\mathbf{U}_{n} \mathbf{U}_{n}^{H}(\operatorname{diag}(\mathbb{S})) \mathbf{v}_{\mathbb{S}}\left(\bar{\theta}_{i}\right) \underbrace{\mathbf{v}_{\mathbb{S}}\left(\bar{\theta}_{i}\right) \mathbf{U}_{n}}_{\mathbf{0}} \mathbf{U}_{n}^{H}) \\
\quad-\operatorname{vec}(\mathbf{U}_{n} \underbrace{\mathbf{U}_{n}^{H} \mathbf{v}_{\mathbb{S}}\left(\bar{\theta}_{i}\right)}_{\mathbf{0}} \mathbf{v}_{\mathbb{S}}^{H}\left(\bar{\theta}_{i}\right)(\operatorname{diag}(\mathbb{S})) \mathbf{U}_{n} \mathbf{U}_{n}^{H})=\mathbf{0},
\end{aligned}
$$

where the property $\left(\mathbf{C}^{T} \otimes \mathbf{A}\right) \operatorname{vec}(\mathbf{B})=\operatorname{vec}(\mathbf{A B C})$ is utilized. Hence $\mathbf{A J}(\operatorname{diag}(\mathbb{D})) \mathbf{V}_{\mathbb{D}}=\mathbf{0}$.

Corollary 5. $\mathbf{W}_{\mathbb{D}}^{H} \mathbf{J}^{H} \mathbf{A} \mathbf{J} \mathbf{W}_{\mathbb{D}}=\left(\operatorname{rank}\left(\mathbf{U}_{n}\right)\right) \mathbf{e}_{D+1} \mathbf{e}_{D+1}^{H}$, where $\mathbf{e}_{D+1}$ is defined in (62).
Proof. For $1 \leq i, j \leq D$, the $(i, j)$ th entry of $\mathbf{W}_{\mathbb{D}}^{H} \mathbf{J}^{H} \mathbf{A} \mathbf{J} \mathbf{W}_{\mathbb{D}}$ can be simplified as

$$
\begin{aligned}
& {\left[\mathbf{W}_{\mathbb{D}}^{H} \mathbf{J}^{H} \mathbf{A} \mathbf{J} \mathbf{W}_{\mathbb{D}}\right]_{i, j} } \\
= & \mathbf{v}_{\mathbb{D}}^{H}\left(\bar{\theta}_{i}\right) \mathbf{J}^{H} \mathbf{A} \mathbf{J}_{\mathbb{D}}\left(\bar{\theta}_{j}\right) \\
= & {\left[\mathbf{v}_{\mathbb{S}}^{T}\left(\bar{\theta}_{i}\right) \otimes \mathbf{v}_{\mathbb{S}}^{H}\left(\bar{\theta}_{i}\right)\right]\left[\left(\mathbf{U}_{n} \mathbf{U}_{n}^{H}\right)^{T} \otimes\left(\mathbf{U}_{n} \mathbf{U}_{n}^{H}\right)\right]\left[\mathbf{v}_{\mathbb{S}}^{*}\left(\bar{\theta}_{j}\right) \otimes \mathbf{v}_{\mathbb{S}}\left(\bar{\theta}_{j}\right)\right] } \\
= & \left(\mathbf{v}_{\mathbb{S}}^{H}\left(\bar{\theta}_{j}\right) \mathbf{U}_{n} \mathbf{U}_{n}^{H} \mathbf{v}_{\mathbb{S}}\left(\bar{\theta}_{i}\right)\right)^{T} \otimes\left(\mathbf{v}_{\mathbb{S}}^{H}\left(\bar{\theta}_{i}\right) \mathbf{U}_{n} \mathbf{U}_{n}^{H} \mathbf{v}_{\mathbb{S}}\left(\bar{\theta}_{j}\right)\right)=0 .
\end{aligned}
$$

The $(i, D+1)$ th entry of $\mathbf{W}_{\mathbb{D}}^{H} \mathbf{J}^{H} \mathbf{A} \mathbf{J} \mathbf{W}_{\mathbb{D}}$ becomes

$$
\begin{aligned}
& {\left[\mathbf{W}_{\mathbb{D}}^{H} \mathbf{J}^{H} \mathbf{A} \mathbf{J} \mathbf{W}_{\mathbb{D}}\right]_{i, D+1} } \\
= & \mathbf{v}_{\mathbb{D}}^{H}\left(\bar{\theta}_{i}\right) \mathbf{J}^{H} \mathbf{A} \mathbf{J} \mathbf{e}_{0} \\
= & {\left[\mathbf{v}_{\mathbb{S}}^{T}\left(\bar{\theta}_{i}\right) \otimes \mathbf{v}_{\mathbb{S}}^{H}\left(\bar{\theta}_{i}\right)\right]\left[\left(\mathbf{U}_{n} \mathbf{U}_{n}^{H}\right)^{T} \otimes\left(\mathbf{U}_{n} \mathbf{U}_{n}^{H}\right)\right] \operatorname{vec}(\mathbf{I}) } \\
= & \operatorname{vec}\left(\mathbf{v}_{\mathbb{S}}^{H}\left(\bar{\theta}_{i}\right) \mathbf{U}_{n} \mathbf{U}_{n}^{H} \mathbf{U}_{n} \mathbf{U}_{n}^{H} \mathbf{v}_{\mathbb{S}}\left(\bar{\theta}_{i}\right)\right)=0
\end{aligned}
$$

Similarly, the $(D+1, j)$ th entry of $\mathbf{W}_{\mathbb{D}}^{H} \mathbf{J}^{H} \mathbf{A} \mathbf{J} \mathbf{W}_{\mathbb{D}}$ is also zero. Finally the $(D+1, D+1)$ th entry of $\mathbf{W}_{\mathbb{D}}^{H} \mathbf{J}^{H} \mathbf{A} \mathbf{J} \mathbf{W}_{\mathbb{D}}$ is given by

$$
\begin{aligned}
& {\left[\mathbf{W}_{\mathbb{D}}^{H} \mathbf{J}^{H} \mathbf{A} \mathbf{J} \mathbf{W}_{\mathbb{D}}\right]_{D+1, D+1} } \\
= & \operatorname{vec}(\mathbf{I})^{H}\left[\left(\mathbf{U}_{n} \mathbf{U}_{n}^{H}\right)^{T} \otimes\left(\mathbf{U}_{n} \mathbf{U}_{n}^{H}\right)\right] \operatorname{vec}(\mathbf{I}) \\
= & \operatorname{tr}\left(\mathbf{U}_{n} \mathbf{U}_{n}^{H} \mathbf{I U}_{n} \mathbf{U}_{n}^{H} \mathbf{I}\right)=\operatorname{rank}\left(\mathbf{U}_{n}\right),
\end{aligned}
$$

since $\operatorname{tr}(\mathbf{A B C D})=\operatorname{vec}\left(\mathbf{B}^{H}\right)^{H}\left(\mathbf{A}^{T} \otimes \mathbf{C}\right) \operatorname{vec}(\mathbf{D})$ and $\operatorname{tr}(\mathbf{A B})=\operatorname{tr}(\mathbf{B A})$.

To evaluate the asymptotic expression of the CRB, we first consider the inverse of $\mathbf{W}_{\mathbb{D}}^{H} \mathbf{M}^{2} \mathbf{W}_{\mathbb{D}}$, based on (C.4) and Corollary 5:

$$
\begin{aligned}
&\left(\mathbf{W}_{\mathbb{D}}^{H} \mathbf{M}^{2} \mathbf{W}_{\mathbb{D}}\right)^{-1} \\
& \stackrel{\text { large } p}{\longrightarrow}\left(\mathbf{W}_{\mathbb{D}}^{H} \mathbf{J}^{H} \mathbf{A} \mathbf{J} \mathbf{W}_{\mathbb{D}}+p^{-1} \mathbf{W}_{\mathbb{D}}^{H} \mathbf{J}^{H} \mathbf{B} \mathbf{J} \mathbf{W}_{\mathbb{D}}\right)^{-1} \\
&=\left(p^{-1} \mathbf{W}_{\mathbb{D}}^{H} \mathbf{J}^{H} \mathbf{B J} \mathbf{W}_{\mathbb{D}}+\operatorname{rank}\left(\mathbf{U}_{n}\right) \mathbf{e}_{D+1} \mathbf{e}_{D+1}^{H}\right)^{-1} \\
&= p\left(\mathbf{W}_{\mathbb{D}}^{H} \mathbf{M}_{\infty}^{2} \mathbf{W}_{\mathbb{D}}+\operatorname{rank}\left(\mathbf{U}_{n}\right) p \times \mathbf{e}_{D+1} \mathbf{e}_{D+1}^{H}\right)^{-1},
\end{aligned}
$$

where $\mathbf{M}_{\infty}$ is the positive definite squared root of $\mathbf{J}^{H} \mathbf{B J}$, as defined in (59).

To be more rigorous, we need to show that $\mathbf{J}^{H} \mathbf{B J}$ is positive semidefinite. Since $\mathbf{U}_{s} \boldsymbol{\Lambda}^{-1} \mathbf{U}_{s}^{H}$ and $\mathbf{U}_{n} \mathbf{U}_{n}^{H}$ are both positive semidefinite, $\left(\mathbf{U}_{s} \boldsymbol{\Lambda}^{-1} \mathbf{U}_{s}^{H}\right)^{T} \otimes\left(\mathbf{U}_{n} \mathbf{U}_{n}^{H}\right)$ and $\left(\mathbf{U}_{n} \mathbf{U}_{n}^{H}\right)^{T} \otimes\left(\mathbf{U}_{s} \boldsymbol{\Lambda}^{-1} \mathbf{U}_{s}^{H}\right)$ are also positive semidefinite [56]. Then, according to (C.2), B is a sum of two positive semidefinite matrices, implying $\mathbf{B}$ is also positive semidefinite [56]. These arguments prove the existence of $\mathbf{M}_{\infty}$.

If $\mathbf{W}_{\mathbb{D}}^{H} \mathbf{M}_{\infty}^{2} \mathbf{W}_{\mathbb{D}}$ has full rank, then applying the matrix inversion lemma [37] to (C.7) leads to

$$
\begin{aligned}
& \stackrel{\left(\mathbf{W}_{\mathbb{D}}^{H} \mathbf{M}^{2} \mathbf{W}_{\mathbb{D}}\right)^{-1}}{\stackrel{\text { large } p}{\longrightarrow}} p\left(\left(\mathbf{W}_{\mathbb{D}}^{H} \mathbf{M}_{\infty}^{2} \mathbf{W}_{\mathbb{D}}\right)^{-1}\right. \\
& \left.-\frac{\left(\mathbf{W}_{\mathbb{D}}^{H} \mathbf{M}_{\infty}^{2} \mathbf{W}_{\mathbb{D}}\right)^{-1} \mathbf{e}_{D+1} \mathbf{e}_{D+1}^{H}\left(\mathbf{W}_{\mathbb{D}}^{H} \mathbf{M}_{\infty}^{2} \mathbf{W}_{\mathbb{D}}\right)^{-1}}{\left(\operatorname{rank}\left(\mathbf{U}_{n}\right) p\right)^{-1}+\mathbf{e}_{D+1}^{H}\left(\mathbf{W}_{\mathbb{D}}^{H} \mathbf{M}_{\infty}^{2} \mathbf{W}_{\mathbb{D}}\right)^{-1} \mathbf{e}_{D+1}}\right)
\end{aligned}
$$




$$
\begin{aligned}
& \stackrel{\text { large } p}{\longrightarrow} p\left(\left(\mathbf{W}_{\mathbb{D}}^{H} \mathbf{M}_{\infty}^{2} \mathbf{W}_{\mathbb{D}}\right)^{-1}\right. \\
& \left.-\frac{\left(\mathbf{W}_{\mathbb{D}}^{H} \mathbf{M}_{\infty}^{2} \mathbf{W}_{\mathbb{D}}\right)^{-1} \mathbf{e}_{D+1} \mathbf{e}_{D+1}^{H}\left(\mathbf{W}_{\mathbb{D}}^{H} \mathbf{M}_{\infty}^{2} \mathbf{W}_{\mathbb{D}}\right)^{-1}}{\mathbf{e}_{D+1}^{H}\left(\mathbf{W}_{\mathbb{D}}^{H} \mathbf{M}_{\infty}^{2} \mathbf{W}_{\mathbb{D}}\right)^{-1} \mathbf{e}_{D+1}}\right),
\end{aligned}
$$

where it is assumed that $\mathbf{e}_{D+1}^{H}\left(\mathbf{W}_{\mathbb{D}}^{H} \mathbf{M}_{\infty}^{2} \mathbf{W}_{\mathbb{D}}\right)^{-1} \mathbf{e}_{D+1}$ is not zero. Next, we consider the asymptotic expression of $\mathbf{G}_{0}^{H} \mathbf{G}_{0}$ for large SNR,

$$
\mathbf{G}_{0}^{H} \mathbf{G}_{0}
$$

$$
\stackrel{\text { large } p}{\longrightarrow} p^{2} \mathbf{V}_{\mathbb{D}}^{H}(\operatorname{diag}(\mathbb{D})) \mathbf{J}^{H}\left(\mathbf{A}+p^{-1} \mathbf{B}\right) \mathbf{J}(\operatorname{diag}(\mathbb{D})) \mathbf{V}_{\mathbb{D}}
$$$$
=p \mathbf{V}_{\mathbb{D}}^{H}(\operatorname{diag}(\mathbb{D})) \mathbf{J}^{H} \mathbf{B J}(\operatorname{diag}(\mathbb{D})) \mathbf{V}_{\mathbb{D}}=p \mathbf{G}_{\infty}^{H} \mathbf{G}_{\infty}, \quad(\mathrm{C} .9)
$$

due to Corollary 4. Here $\mathbf{G}_{\infty}$ is defined in (60). Similarly, $\left(\mathbf{M W}_{\mathbb{D}}\right)^{H} \mathbf{G}_{0}$ has an asymptotic expression,

$$
\begin{aligned}
\left(\mathbf{M W}_{\mathbb{D}}\right)^{H} \mathbf{G}_{0} & \stackrel{\text { large } p}{\longrightarrow} \mathbf{W}_{\mathbb{D}}^{H} \mathbf{J}^{H}\left(\mathbf{A}+p^{-1} \mathbf{B}\right) \mathbf{J}(\operatorname{diag}(\mathbb{D})) \mathbf{V}_{\mathbb{D}}(p \mathbf{I}) \\
& =\left(\mathbf{M}_{\infty} \mathbf{W}_{\mathbb{D}}\right)^{H} \mathbf{G}_{\infty}
\end{aligned}
$$

Substituting (C.8), (C.9), and (C.10) into (49) yields (57).

\section{Appendix C.2. Proof of Theorem 4}

Since $\mathbf{V}_{\mathbb{S}}$ has full row rank, $\mathbf{V}_{\mathbb{S}} \mathbf{V}_{\mathbb{S}}^{H}$ is nonsingular. It can be inferred from (52) that

$$
\begin{aligned}
\mathbf{R}_{\mathbb{S}}^{-1} \stackrel{\text { large } p}{\longrightarrow}\left(p \mathbf{V}_{\mathbb{S}} \mathbf{V}_{\mathbb{S}}^{H}\right)^{-1}, \\
\mathbf{M} \stackrel{\text { large } p}{\longrightarrow}\left(\mathbf{J}^{H}\left(\left(p \mathbf{V}_{\mathbb{S}} \mathbf{V}_{\mathbb{S}}^{H}\right)^{-T} \otimes\left(p \mathbf{V}_{\mathbb{S}} \mathbf{V}_{\mathbb{S}}^{H}\right)^{-1}\right) \mathbf{J}\right)^{\frac{1}{2}}=\frac{\mathbf{M}_{\infty}}{p}, \\
\quad \mathbf{G} \stackrel{\text { large } p}{\longrightarrow} \frac{\mathbf{M}_{\infty}}{p}(\operatorname{diag}(\mathbb{D})) \mathbf{V}_{\mathbb{D}}(p \mathbf{I})=\mathbf{G}_{\infty}
\end{aligned}
$$

Replacing $\mathbf{M}$ and $\mathbf{G}_{0}$ with their limits in (49) proves this property.

\section{Appendix D. Proof of Theorem 5}

To prove that $\operatorname{rank}\left(\mathbf{A}_{c}\right)=2 D+1$, it suffices to show that, there exists a $(2 D+1) \times(2 D+1)$ full rank submatrix. Since $D \leq(|\mathbb{U}|-1) / 2$, the following matrix $\mathbf{S}_{0}$ is a submatrix of $\mathbf{A}_{c}$,

$$
\mathbf{S}_{0}=\left[\begin{array}{ccccccc}
-D \alpha_{1}^{-D} & \ldots & -D \alpha_{D}^{-D} & \alpha_{1}^{-D} & \ldots & \alpha_{D}^{-D} & 0 \\
\vdots & \ddots & \vdots & \vdots & \ddots & \vdots & \vdots \\
-2 \alpha_{1}^{-2} & \ldots & -2 \alpha_{D}^{-2} & \alpha_{D}^{-2} & \ldots & \alpha_{D}^{-2} & 0 \\
-\alpha_{1}^{-1} & \ldots & -\alpha_{D}^{-1} & \alpha_{1}^{-1} & \ldots & \alpha_{D}^{-1} & 0 \\
0 & \ldots & 0 & 1 & \ldots & 1 & 1 \\
\alpha_{1} & \ldots & \alpha_{D} & \alpha_{1} & \ldots & \alpha_{D} & 0 \\
2 \alpha_{1}^{2} & \ldots & 2 \alpha_{D}^{2} & \alpha_{1}^{2} & \ldots & \alpha_{D}^{2} & 0 \\
\vdots & \ddots & \vdots & \vdots & \ddots & \vdots & \vdots \\
D \alpha_{1}^{D} & \ldots & D \alpha_{D}^{D} & \alpha_{1}^{D} & \ldots & \alpha_{D}^{D} & 0
\end{array}\right],
$$

where $\alpha_{i}=e^{j 2 \pi \bar{\theta}_{i}}$ for $i=1,2, \ldots, D$. It will be shown that $\operatorname{rank}\left(\mathbf{S}_{0}\right)=2 D+1$. Consider another matrix $\mathbf{S}_{1}$, defined as

$$
\mathbf{S}_{1}=\mathbf{S}_{0} \underbrace{\left[\begin{array}{ccc}
\mathbf{L}_{1,1} & \mathbf{0} & \mathbf{0} \\
\mathbf{L}_{2,1} & \mathbf{L}_{2,2} & \mathbf{0} \\
\mathbf{0}^{T} & \mathbf{0}^{T} & 1
\end{array}\right]}_{\mathbf{L}},
$$

where the matrices $\mathbf{L}_{1,1}, \mathbf{L}_{2,1}$, and $\mathbf{L}_{2,2}$ are given by

$$
\begin{aligned}
& \mathbf{L}_{1,1}=\operatorname{diag}\left(\alpha_{1}^{D-1}, \alpha_{2}^{D-1}, \ldots, \alpha_{D}^{D-1}\right), \\
& \mathbf{L}_{2,1}=\operatorname{diag}\left(D \alpha_{1}^{D-1}, D \alpha_{2}^{D-1}, \ldots, D \alpha_{D}^{D-1}\right), \\
& \mathbf{L}_{2,2}=\operatorname{diag}\left(\alpha_{1}^{D}, \alpha_{2}^{D}, \ldots, \alpha_{D}^{D}\right) .
\end{aligned}
$$

$\mathbf{S}_{0}, \mathbf{S}_{1}$, and $\mathbf{L}$ are square matrices of size $2 D+1$. Expanding (D.1) results in

$\mathbf{S}_{1}=$

$$
\left[\begin{array}{ccccccc}
0 & \ldots & 0 & 1 & \ldots & 1 & 0 \\
1 & \ldots & 1 & \alpha_{1} & \ldots & \alpha_{D} & 0 \\
2 \alpha_{1} & \ldots & 2 \alpha_{D} & \alpha_{1}^{2} & \ldots & \alpha_{D}^{2} & 0 \\
\vdots & \ddots & \vdots & \vdots & \ddots & \vdots & \vdots \\
(D-1) \alpha_{1}^{D-2} & \ldots & (D-1) \alpha_{D}^{D-2} & \alpha_{1}^{D-1} & \ldots & \alpha_{D}^{D-1} & 0 \\
D \alpha_{1}^{D-1} & \ldots & D \alpha_{D}^{D-1} & \alpha_{1}^{D} & \ldots & \alpha_{D}^{D} & 1 \\
(D+1) \alpha_{1}^{D} & \ldots & (D+1) \alpha_{D}^{D} & \alpha_{1}^{D+1} & \ldots & \alpha_{D}^{D+1} & 0 \\
\vdots & \ddots & \vdots & \vdots & \ddots & \vdots & \vdots \\
2 D \alpha_{1}^{2 D-1} & \ldots & 2 D \alpha_{D}^{2 D-1} & \alpha_{1}^{2 D} & \ldots & \alpha_{D}^{2 D} & 0
\end{array}\right] .
$$

It holds true that $\operatorname{rank}\left(\mathbf{S}_{0}\right)=\operatorname{rank}\left(\mathbf{S}_{1}\right)$, since the lower triangular matrix $\mathbf{L}$ has non-zero diagonal entries.

Next, it will be shown that $\mathbf{S}_{1}$ has full rank. Let $\mathbf{h}=$ $\left[h_{0}, h_{1}, \ldots, h_{2 D}\right]^{T}$ satisfy $\mathbf{h}^{T} \mathbf{S}_{1}=\mathbf{0}$. Define a polynomial $H(z)=\sum_{n=0}^{2 D} h_{n} z^{n}$. Then $\mathbf{h}^{T} \mathbf{S}_{1}=\mathbf{0}$ is equivalent to

$$
\begin{gathered}
H^{\prime}\left(\alpha_{1}\right)=\cdots=H^{\prime}\left(\alpha_{D}\right)=0, \\
H\left(\alpha_{1}\right)=\cdots=H\left(\alpha_{D}\right)=0, \\
h_{D}=0,
\end{gathered}
$$

where $H^{\prime}(z)=(\mathrm{d} / \mathrm{d} z) H(z)=\sum_{n=0}^{2 D} h_{n}\left(n z^{n-1}\right)$. Since the DOAs are distinct, (D.2) and (D.3) indicate $H(z)$ can be expressed as

$$
H(z)=K \prod_{i=1}^{D}\left(z-\alpha_{i}\right)^{2}=K_{0} z^{D} \underbrace{\prod_{i=1}^{D}\left(\frac{\alpha_{i}}{z}-2+\frac{z}{\alpha_{i}}\right)}_{G(z)},
$$

where $K_{0}=K \prod_{i=1}^{D} \alpha_{i}$ for some constant $K$. Eqs. (D.4) and (D.5) imply

$$
\begin{aligned}
0 & =K_{0} \times(\text { Constant term of } G(z)) \\
& =K_{0} \int_{-\frac{1}{2}}^{\frac{1}{2}} G\left(e^{j 2 \pi \bar{\theta}}\right) \mathrm{d} \bar{\theta} \\
& =K_{0} \int_{-\frac{1}{2}}^{\frac{1}{2}} \prod_{i=1}^{D}\left(e^{-j 2 \pi\left(\bar{\theta}-\bar{\theta}_{i}\right)}-2+e^{j 2 \pi\left(\bar{\theta}-\bar{\theta}_{i}\right)}\right) \mathrm{d} \bar{\theta},
\end{aligned}
$$


where the constant term is based on the inverse discretetime Fourier transform of $G\left(e^{j 2 \pi \bar{\theta}}\right)[97]$. (D.6) can be simplified as

$$
0=K_{0}(-2)^{D} \overbrace{\int_{-\frac{1}{2}}^{\frac{1}{2}} \underbrace{\left(\prod_{i=1}^{D}\left(1-\cos 2 \pi\left(\bar{\theta}-\bar{\theta}_{i}\right)\right)\right)}_{\geq 0} \mathrm{~d} \bar{\theta}}^{00} .
$$

Since the integrand is non-negative, this integral is strictly positive, implying $K_{0}=K=0$ and $\mathbf{h}=\mathbf{0}$. Hence, $2 D+$ $1=\operatorname{rank}\left(\mathbf{S}_{1}\right)=\operatorname{rank}\left(\mathbf{S}_{0}\right)=\operatorname{rank}\left(\mathbf{A}_{c}\right)$.

\section{References}

[1] Y. Abramovich, D. Gray, A. Gorokhov, N. Spencer, Positivedefinite Toeplitz completion in DOA estimation for nonuniform linear antenna arrays - Part I: Fully augmentable arrays, IEEE Trans. Signal Proc. 46 (9) (1998) 2458-2471.

[2] Y. Abramovich, N. Spencer, A. Gorokhov, Positive-definite Toeplitz completion in DOA estimation for nonuniform linear antenna arrays - Part II: Partially augmentable arrays, IEEE Trans. Signal Proc. 47 (6) (1999) 1502-1521.

[3] K. Adhikari, J. R. Buck, K. E. Wage, Extending coprime sensor arrays to achieve the peak side lobe height of a full uniform linear array, EURASIP J. Adv. Signal Process. 2014 (1).

[4] J. Arsac, Nouveau reseau pour l'observation radioastronomique de la brillance sur le soleil a $9350 \mathrm{mc} / \mathrm{s}$, Compt. Rend. Acad. Sci. 240 (1955) 942-945.

[5] M. Atkinson, N. Santoro, J. Urrutia, Integer sets with distinct sums and differences and carrier frequency assignments for nonlinear repeaters, IEEE Trans. Commun. 34 (6) (1986) 614-617.

[6] A. Barabell, Improving the resolution performance of eigenstructure-based direction-finding algorithms, in: Proc. IEEE Int. Conf. Acoust., Speech, and Sig. Proc., vol. 8, 1983, pp. 336-339.

[7] A. J. Barabell, J. Capon, D. F. DeLong, J. R. Johnson, K. D. Senne, Performance comparison of superresolution array processing algorithms, Tech. rep., MIT Lincoln Laboratory, Lexington, MA (1984, revised 1998).

[8] M. S. Bartlett, Smoothing periodograms from time-series with continuous spectra, Nature 161 (1948) 686-687.

[9] S. Bedrosian, Nonuniform linear arrays: Graph-theoretic approach to minimum redundancy, Proc. IEEE 74 (7) (1986) 1040-1043.

[10] G. Bienvenu, L. Kopp, Optimality of high resolution array processing using the eigensystem approach, IEEE Trans. Acoust., Speech, Signal Proc. 31 (5) (1983) 1235-1248.

[11] G. Bouleux, P. Stoica, R. Boyer, An optimal PriorknowLEDGE-based DOA estimation method, in: Proc. European Signal Proc. Conf., 2009, pp. 869-873.

[12] R. N. Bracewell, Radio astronomy techniques, vol. 54, Berlin: Springer, 1962, pp. 42-129.

[13] R. N. Bracewell, Optimum spacings for radio telescopes with unfilled apertures, Nat. Acad. Sci. Nat. Res. Counc., Publ. 1408 (1966) 243-244.

[14] A. Camps, A. Cardama, D. Infantes, Synthesis of large lowredundancy linear arrays, IEEE Trans. Antennas Propag. 49 (12) (2001) 1881-1883.

[15] J. Capon, High-resolution frequency-wavenumber spectrum analysis, Proc. IEEE 57 (8) (1969) 1408-1418.

[16] C. Chambers, T. Tozer, K. Sharman, T. Durrani, Temporal and spatial sampling influence on the estimates of superimposed narrowband signals: when less can mean more, IEEE Trans. Signal Proc. 44 (12) (1996) 3085-3098.
[17] J. Chen, Q. Liang, B. Z. X. Wu, Spectrum efficiency of nested sparse sampling and coprime sampling, EURASIP Journal on Wireless Communications and Networking 2013 (47).

[18] P. Chevalier, L. Albera, A. Ferreol, P. Comon, On the virtual array concept for higher order array processing, IEEE Trans. Signal Proc. 53 (4) (2005) 1254-1271.

[19] P. Chevalier, A. Ferreol, On the virtual array concept for the fourth-order direction finding problem, IEEE Trans. Signal Proc. 47 (9) (1999) 2592-2595.

[20] H. Cramér, Mathematical Methods of Statistics, Princeton University Press, 1946.

[21] M. Dogan, J. Mendel, Applications of cumulants to array processing-Part I: Aperture extension and array calibration, IEEE Trans. Signal Proc. 43 (5) (1995) 1200-1216.

[22] A. Dollas, W. Rankin, D. McCracken, A new algorithm for Golomb ruler derivation and proof of the 19 mark ruler, IEEE Trans. Inf. Theory 44 (1) (1998) 379-382.

[23] J. Dong, Q. Li, R. Jin, Y. Zhu, Q. Huang, L. Gui, A method for seeking low-redundancy large linear arrays in aperture synthesis microwave radiometers, IEEE Trans. Antennas Propag. 58 (6) (2010) 1913-1921.

[24] E. Evans, J. R. Johnson, D. F. Sun, High resolution angular spectrum estimation techniques for terrain scattering analysis and angle of arrival estimation, in: Proc. 1st ASSP Workshop Spectral Estimation, Hamilton, Ontario, Canada, 1981, pp. 134-139.

[25] L. Godara, Application of antenna arrays to mobile communications, Part II: Beam-forming and direction-of-arrival considerations, Proc. IEEE 85 (8) (1997) 1195-1245.

[26] J. R. Guerci, Space-Time Adaptive Processing for Radar, Artech House, 2003.

[27] M. Haardt, J. Nossek, Unitary ESPRIT: how to obtain increased estimation accuracy with a reduced computational burden, IEEE Trans. Signal Proc. 43 (5) (1995) 1232-1242.

[28] K. Han, A. Nehorai, Improved source number detection and direction estimation with nested arrays and ULAs using jackknifing, IEEE Trans. Signal Proc. 61 (23) (2013) 6118-6128.

[29] K. Han, A. Nehorai, Wideband Gaussian source processing using a linear nested array, IEEE Signal Proc. Lett. 20 (11) (2013) 1110-1113.

[30] K. Han, A. Nehorai, Nested array processing for distributed sources, IEEE Signal Proc. Lett. 21 (9) (2014) 1111-1114.

[31] K. Han, A. Nehorai, Nested vector-sensor array processing via tensor modeling, IEEE Trans. Signal Proc. 62 (10) (2014) $2542-2553$

[32] K. Han, P. Yang, A. Nehorai, Calibrating nested sensor arrays with model errors, IEEE Trans. Antennas Propag. 63 (11) (2015) 4739-4748.

[33] S. Haykin, Array Signal Processing, Prentice-Hall, 1984.

[34] S. Haykin, J. Reilly, V. Kezys, E. Vertatschitsch, Some aspects of array signal processing, Proc. Inst. Electr. Eng. F-Radar Signal Process. 139 (1) (1992) 1-26.

[35] Z.-Q. He, Z.-P. Shia, L. Huang, Covariance sparsity-aware DOA estimation for nonuniform noise, Digital Signal Processing 28 (2014) 75-81.

[36] R. Hoctor, S. Kassam, The unifying role of the coarray in aperture synthesis for coherent and incoherent imaging, Proc. IEEE 78 (4) (1990) 735-752.

[37] R. A. Horn, C. R. Johnson, Matrix Analysis, Cambridge, 1985.

[38] P.-C. Hsiao, Y.-T. Lin, S.-G. Chen, An efficient searching algorithm for high DoF non-uniform linear antenna array with constrained spacing, in: 2015 13th International Conference on Telecommunications (ConTEL), 2015, pp. 1-5.

[39] M. Ishiguro, Minimum redundancy linear arrays for a large number of antennas, Radio Science 15 (6) (1980) 1163-1170.

[40] M. Jansson, B. Goransson, B. Ottersten, A subspace method for direction of arrival estimation of uncorrelated emitter signals, IEEE Trans. Signal Proc. 47 (4) (1999) 945-956.

[41] M. Jansson, B. Ottersten, Structured covariance matrix estimation: a parametric approach, in: Proc. IEEE Int. Conf. Acoust., Speech, and Sig. Proc., vol. 5, 2000, pp. 3172-3175 
vol.5.

[42] S. M. Kay, Fundamentals of Statistical Signal Proceesing: Estimation Theory, Prentice Hall PTR, 1993.

[43] R. Klemm, Space-Time Adaptive Processing: Principles and Applications, IEEE Press, 1998.

[44] A. Koochakzadeh, P. Pal, On the robustness of co-prime sampling, in: Proc. European Signal Proc. Conf., 2015.

[45] A. Koochakzadeh, P. Pal, Sparse source localization in presence of co-array perturbations, in: Sampling Theory and Applications (SampTA), Washington D.C., USA, 2015.

[46] H. Krim, M. Viberg, Two decades of array signal processing research: the parametric approach, IEEE Signal Process. Mag. 13 (4) (1996) 67-94.

[47] A. Lam, D. Sarwate, On optimum time-hopping patterns, IEEE Trans. Commun. 36 (3) (1988) 380-382.

[48] J. Leech, On the representation of $1,2, \ldots, n$ by differences, J. London Math. Soc. 1 (2) (1956) 160-169.

[49] H. Li, P. Stoica, J. Li, Computationally efficient maximum likelihood estimation of structured covariance matrices, IEEE Trans. Signal Proc. 47 (5) (1999) 1314-1323.

[50] D. Linebarger, I. Sudborough, I. Tollis, Difference bases and sparse sensor arrays, IEEE Trans. Inf. Theory 39 (2) (1993) $716-721$.

[51] C.-L. Liu, P. P. Vaidyanathan, Super nested arrays: Linear sparse arrays with reduced mutual coupling - Part I: Fundamentals, submitted to IEEE Trans. Signal Proc.

[52] C.-L. Liu, P. P. Vaidyanathan, Super nested arrays: Linear sparse arrays with reduced mutual coupling - Part II: Highorder extensions, submitted to IEEE Trans. Signal Proc.

[53] C.-L. Liu, P. P. Vaidyanathan, Remarks on the spatial smoothing step in coarray MUSIC, IEEE Signal Proc. Lett. 22 (9) (2015) 1438-1442.

[54] C.-L. Liu, P. P. Vaidyanathan, Super nested arrays: Sparse arrays with less mutual coupling than nested arrays, in: Proc. IEEE Int. Conf. Acoust., Speech, and Sig. Proc., 2016.

[55] C.-L. Liu, P. P. Vaidyanathan, P. Pal, Coprime coarray interpolation for DOA estimation via nuclear norm minimization, in: Proc. IEEE Int. Symp. Circuits and Syst., 2016.

[56] H. Lütkepohl, Handbook of Matrices, John Wiley \& Sons, 1996.

[57] W.-K. Ma, T.-H. Hsieh, C.-Y. Chi, DOA estimation of quasistationary signals via Khatri-Rao subspace, in: Proc. IEEE Int. Conf. Acoust., Speech, and Sig. Proc., 2009, pp. 21652168 .

[58] W.-K. Ma, T.-H. Hsieh, C.-Y. Chi, DOA estimation of quasistationary signals with less sensors than sources and unknown spatial noise covariance: A Khatri-Rao subspace approach, IEEE Trans. Signal Proc. 58 (4) (2010) 2168-2180.

[59] A. Manikas, Differential Geometry In Array Processing, Imperial College Press, 2004.

[60] D. G. Manolakis, V. K. Ingle, S. M. Kogon, Statistical and Adaptive Signal Processing: Spectral Estimation, Signal Modeling, Adaptive Filtering, and Array Processing, Artech House, 2005.

[61] A. T. Moffet, Minimum-redundancy linear arrays, IEEE Trans. Antennas Propag. 16 (2) (1968) 172-175.

[62] B. Ottersten, M. Viberg, T. Kailath, Performance analysis of the total least squares ESPRIT algorithm, IEEE Trans. Signal Proc. 39 (5) (1991) 1122-1135.

[63] P. Pal, P. P. Vaidyanathan, Nested arrays: A novel approach to array processing with enhanced degrees of freedom, IEEE Trans. Signal Proc. 58 (8) (2010) 4167-4181.

[64] P. Pal, P. P. Vaidyanathan, Coprime sampling and the MUSIC algorithm, in: Proc. IEEE Dig. Signal Proc. Signal Proc. Educ. Workshop, 2011, pp. 289-294.

[65] P. Pal, P. P. Vaidyanathan, Correlation-aware techniques for sparse support recovery, in: Proc. IEEE Statist. Signal Process. (SSP) Workshop, 2012, pp. 53-56.

[66] P. Pal, P. P. Vaidyanathan, Multiple level nested array: An efficient geometry for $2 q$ th order cumulant based array processing, IEEE Trans. Signal Proc. 60 (3) (2012) 1253-1269.
[67] P. Pal, P. P. Vaidyanathan, Pushing the limits of sparse support recovery using correlation information, IEEE Trans. Signal Proc. 63 (3) (2015) 711-726.

[68] D. Pearson, S. Pillai, Y. Lee, An algorithm for near-optimal placement of sensor elements, IEEE Trans. Inf. Theory 36 (6) (1990) 1280-1284.

[69] M. Pesavento, A. Gershman, M. Haardt, Unitary root-MUSIC with a real-valued eigendecomposition: a theoretical and experimental performance study, IEEE Trans. Signal Proc. 48 (5) (2000) 1306-1314.

[70] S. Pillai, Y. Bar-Ness, F. Haber, A new approach to array geometry for improved spatial spectrum estimation, Proc. IEEE 73 (10) (1985) 1522-1524.

[71] S. Pillai, F. Haber, Statistical analysis of a high resolution spatial spectrum estimator utilizing an augmented covariance matrix, IEEE Trans. Acoust., Speech, Signal Proc. 35 (11) (1987) 1517-1523.

[72] V. F. Pisarenko, The retrieval of harmonics from a covariance function, Geophys. J. R. astr. Soc. 33 (1973) 347-366.

[73] B. Porat, B. Friedlander, Analysis of the asymptotic relative efficiency of the MUSIC algorithm, IEEE Trans. Acoust., Speech, Signal Proc. 36 (4) (1988) 532-544.

[74] B. Porat, B. Friedlander, Direction finding algorithms based on high-order statistics, IEEE Trans. Signal Proc. 39 (9) (1991) 2016-2024.

[75] S. Qin, Y. Zhang, M. Amin, Generalized coprime array configurations, in: Proc. IEEE Sensor Array and Multichannel Signal Processing Workshop, 2014, pp. 529-532.

[76] S. Qin, Y. Zhang, M. Amin, Generalized coprime array configurations for direction-of-arrival estimation, IEEE Trans. Signal Proc. 63 (6) (2015) 1377-1390.

[77] J. Ramirez, J. Odom, J. Krolik, Exploiting array motion for augmentation of co-prime arrays, in: Proc. IEEE Sensor Array and Multichannel Signal Processing Workshop, 2014, pp. 525528 .

[78] C. R. Rao, Information and the accuracy attainable in the estimation of statistical parameters, Bulletin of the Calcutta Mathematical Society 37 (1945) 81-89.

[79] S. Reddi, Multiple source location: A digital approach, IEEE Trans. Aerosp. Electron. Syst. AES-15 (1) (1979) 95-105.

[80] J. Robinson, A. Bernstein, A class of binary recurrent codes with limited error propagation, IEEE Trans. Inf. Theory 13 (1) (1967) 106-113.

[81] R. Roy, T. Kailath, ESPRIT-estimation of signal parameters via rotational invariance techniques, IEEE Trans. Acoust., Speech, Signal Proc. 37 (7) (1989) 984-995.

[82] M. Rubsamen, A. Gershman, Sparse array design for azimuthal direction-of-arrival estimation, IEEE Trans. Signal Proc. 59 (12) (2011) 5957-5969.

[83] C. Ruf, Numerical annealing of low-redundancy linear arrays, IEEE Trans. Antennas Propag. 41 (1) (1993) 85-90.

[84] R. Schmidt, Multiple emitter location and signal parameter estimation, IEEE Trans. Antennas Propag. 34 (3) (1986) 276280.

[85] Q. Shen, W. Liu, W. Cui, S. Wu, Extension of co-prime arrays based on the fourth-order difference co-array concept, IEEE Signal Proc. Lett. 23 (5) (2016) 615-619.

[86] Q. Shen, W. Liu, W. Cui, S. Wu, Extension of nested arrays with the fourth-order difference co-array enhancement, in: Proc. IEEE Int. Conf. Acoust., Speech, and Sig. Proc., 2016.

[87] M. I. Skolnik, Introduction to Radar Systems, 3rd ed., McGraw Hill, 2001.

[88] P. Stoica, E. Larsson, A. Gershman, The stochastic CRB for array processing: a textbook derivation, IEEE Signal Proc. Lett. 8 (5) (2001) 148-150.

[89] P. Stoica, R. Moses, Spectral Analysis of Signals, Prentice Hall, Upper Saddle River, New Jersey, 2005.

[90] P. Stoica, A. Nehorai, MUSIC, maximum likelihood, and Cramer-Rao bound, IEEE Trans. Acoust., Speech, Signal Proc. 37 (5) (1989) 720-741. 
[91] P. Stoica, A. Nehorai, Performance study of conditional and unconditional direction-of-arrival estimation, IEEE Trans. Acoust., Speech, Signal Proc. 38 (10) (1990) 1783-1795.

[92] M. B. Sverdlik, Optimal Discrete Signals, Sovietsckoe Radio, Moscow, Russia, 1975.

[93] Z. Tan, Y. Eldar, A. Nehorai, Direction of arrival estimation using co-prime arrays: A super resolution viewpoint, IEEE Trans. Signal Proc. 62 (21) (2014) 5565-5576.

[94] H. Taylor, S. W. Golomb, Rulers, Part I, Tech. Rep. 85-05-01, Univ. Southern Calif., Los Angeles (1985).

[95] T. E. Tuncer, B. Friedlander, Classical and Modern Directionof-Arrival Estimation, Academic Press, 2009.

[96] T. E. Tuncer, T. K. Yasar, B. Friedlander, Direction of arrival estimation for nonuniform linear arrays by using array interpolation, Radio Science 42 (4).

[97] P. P. Vaidyanathan, Multirate Systems And Filter Banks, Pearson Prentice Hall, 1993.

[98] P. P. Vaidyanathan, P. Pal, Sparse sensing with co-prime samplers and arrays, IEEE Trans. Signal Proc. 59 (2) (2011) 573586.

[99] P. P. Vaidyanathan, P. Pal, Direct-MUSIC on sparse arrays, in: 2012 International Conference on Signal Processing and Communications (SPCOM), 2012.

[100] P. P. Vaidyanathan, P. Pal, Why does direct-MUSIC on sparsearrays work?, in: Proc. IEEE Asil. Conf. on Sig., Sys., and Comp., 2013, pp. 2007-2011.

[101] P. P. Vaidyanathan, S.-M. Phoong, Y.-P. Lin, Signal Processing and Optimization for Transceiver Systems, Cambridge, 2010.

[102] H. L. Van Trees, Optimum Array Processing: Part IV of Detection, Estimation, and Modulation Theory, Wiley Interscience, 2002.

[103] E. Vertatschitsch, S. Haykin, Nonredundant arrays, Proc. IEEE 74 (1) (1986) 217-217.

[104] P. Wirfält, M. Jansson, G. Bouleux, P. Stoica, Prior knowledge-based direction of arrival estimation, in: Proc. IEEE Int. Conf. Acoust., Speech, and Sig. Proc., 2011, pp. 2540-2543.

[105] P. Wirfält, G. Bouleux, M. Jansson, P. Stoica, Optimal prior knowledge-based direction of arrival estimation, Signal Processing, IET 6 (8) (2012) 731-742.

[106] M. Yang, A. Haimovich, B. Chen, X. Yuan, A new array geometry for DOA estimation with enhanced degrees of freedom, in: Proc. IEEE Int. Conf. Acoust., Speech, and Sig. Proc., 2016.

[107] Y. Zhang, M. Amin, B. Himed, Sparsity-based DOA estimation using co-prime arrays, in: Proc. IEEE Int. Conf. Acoust., Speech, and Sig. Proc., 2013, pp. 3967-3971.

[108] Y. Zhang, S. Qin, M. Amin, DOA estimation exploiting coprime arrays with sparse sensor spacing, in: Proc. IEEE Int. Conf. Acoust., Speech, and Sig. Proc., 2014, pp. 2267-2271. 